\title{
Tolman lengths and rigidity constants of multicomponent fluids: Fundamental theory and numerical examples ${ }^{\circ}$
}

Cite as: J. Chem. Phys. 148, 204702 (2018); https://doi.org/10.1063/1.5026747

Submitted: 23 February 2018. Accepted: 02 May 2018. Published Online: 23 May 2018

Ailo Aasen (D), Edgar M. Blokhuis (D), and Øivind Wilhelmsen

COLLECTIONS

EP This paper was selected as an Editor's Pick
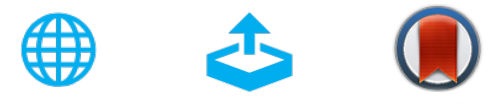

\section{ARTICLES YOU MAY BE INTERESTED IN}

Surface tension of droplets and Tolman lengths of real substances and mixtures from density functional theory

The Journal of Chemical Physics 148, 164703 (2018); https://doi.org/10.1063/1.5020421

A molecular dynamics investigation of the surface tension of water nanodroplets and a new technique for local pressure determination through density correlation

The Journal of Chemical Physics 148, 144503 (2018); https://doi.org/10.1063/1.5004985

The Statistical Mechanical Theory of Surface Tension

The Journal of Chemical Physics 17, 338 (1949); https://doi.org/10.1063/1.1747248

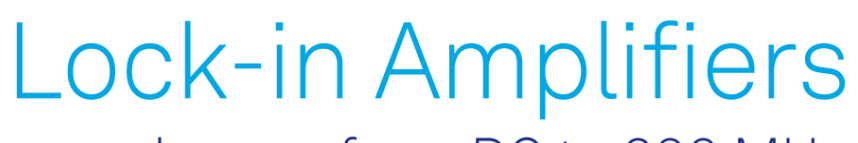

... and more, from DC to $600 \mathrm{MHz}$ Watch 


\title{
Tolman lengths and rigidity constants of multicomponent fluids: Fundamental theory and numerical examples
}

\author{
Ailo Aasen, ${ }^{1,2, a)}$ Edgar M. Blokhuis, ${ }^{3}$ and Øivind Wilhelmsen ${ }^{1,2}$ \\ ${ }^{1}$ Department of Energy and Process Engineering, Norwegian University of Science and Technology, \\ NO-7491 Trondheim, Norway \\ ${ }^{2}$ SINTEF Energy Research, NO-7465 Trondheim, Norway \\ ${ }^{3}$ Colloid and Interface Science, Leiden Institute of Chemistry, 2300 RA Leiden, The Netherlands
}

(Received 23 February 2018; accepted 2 May 2018; published online 23 May 2018)

\begin{abstract}
The curvature dependence of the surface tension can be described by the Tolman length (first-order correction) and the rigidity constants (second-order corrections) through the Helfrich expansion. We present and explain the general theory for this dependence for multicomponent fluids and calculate the Tolman length and rigidity constants for a hexane-heptane mixture by use of square gradient theory. We show that the Tolman length of multicomponent fluids is independent of the choice of dividing surface and present simple formulae that capture the change in the rigidity constants for different choices of dividing surface. For multicomponent fluids, the Tolman length, the rigidity constants, and the accuracy of the Helfrich expansion depend on the choice of path in composition and pressure space along which droplets and bubbles are considered. For the hexane-heptane mixture, we find that the most accurate choice of path is the direction of constant liquid-phase composition. For this path, the Tolman length and rigidity constants are nearly linear in the mole fraction of the liquid phase, and the Helfrich expansion represents the surface tension of hexane-heptane droplets and bubbles within $0.1 \%$ down to radii of $3 \mathrm{~nm}$. The presented framework is applicable to a wide range of fluid mixtures and can be used to accurately represent the surface tension of nanoscopic bubbles and droplets. Published by AIP Publishing. https://doi.org/10.1063/1.5026747
\end{abstract}

\section{INTRODUCTION}

A highly debated question has been how the surface tension of a one-component droplet depends on its radius $R$, or equivalently its total curvature $2 / R \cdot{ }^{1-8}$ The posthumously published landmark paper by Tolman ${ }^{9}$ discussed the firstorder correction of the planar surface tension with respect to curvature,

$$
\sigma^{\mathrm{s}}(R)=\sigma_{0}-\frac{2 \sigma_{0} \delta}{R}+\mathcal{O}\left(\frac{1}{R^{2}}\right)
$$

Here $\sigma^{\mathrm{s}}(R)$ is the surface tension of a spherical droplet (superscript s), $\sigma_{0}=\sigma^{\mathrm{s}}(\infty)$ denotes the planar surface tension, and $\delta$ is referred to as the Tolman length. The sign of the Tolman length for common fluids such as water and the Lennard-Jones (LJ) fluid has been discussed extensively in the literature. ${ }^{3,10-13}$ For the LJ fluid, there is now a consensus that the Tolman length is negative. ${ }^{11,12}$ The sign will, however, depend on the specifics of the system studied; for example, Tröster and Binder ${ }^{14}$ found a positive Tolman length from simulations of a 3D triplet spin Ising lattice model.

Going one step further and incorporating second-order curvature corrections to the surface tension was done by Helfrich, ${ }^{15}$ who introduced the bending rigidity $k$ and the Gaussian rigidity $\bar{k}$ in his study of how the surface tension of elastic membranes depends on curvature. The Helfrich expansion is a second-order Taylor expansion of the surface tension

a)Electronic mail: ailo.aasen@ntnu.no with respect to the interfacial curvatures, which for a spherical interface is given by

$$
\sigma^{\mathrm{s}}(R)=\sigma_{0}-\frac{2 \sigma_{0} \delta}{R}+\frac{2 k+\bar{k}}{R^{2}}+\mathcal{O}\left(\frac{1}{R^{3}}\right),
$$

and for a cylindrical interface (superscript c)

$$
\sigma^{\mathrm{c}}(R)=\sigma_{0}-\frac{\sigma_{0} \delta}{R}+\frac{k}{2 R^{2}}+\mathcal{O}\left(\frac{1}{R^{3}}\right) .
$$

A comparison of Eqs. (2) and (3) shows that it is sufficient to evaluate the Helfrich expansion for a spherical and cylindrical geometry to uniquely determine the Tolman length and both rigidity constants. The Tolman length and the rigidity constants will in this work be referred to as the Helfrich coefficients. Once the Helfrich coefficients are known, the curvature correction for the surface tension of other surface geometries, such as curved films ${ }^{16}$ or gravity-deformed droplets, ${ }^{17}$ can be obtained to second order. The curvature expansions are equally valid for droplets and bubbles. Since the curvature is conventionally taken to be with respect to the liquid phase, the curvature (and hence the radius) is positive for a droplet and negative for a bubble in the curvature expansions.

The physical significance of the rigidity constants for droplets has been debated. ${ }^{10,18}$ In systems with long-range interactions, the second-derivative of $\sigma$ with respect to curvature may not exist, thus invalidating the expansion in Eq. (2); however, for systems with short-range interactions they definitely exist. ${ }^{11}$ For Density Functional Theory (DFT) models, 
the surface tension as a function of curvature is accurately represented by a second-order expansion down to nano-sized droplets. Previous papers have demonstrated this for several one-component fluid models. ${ }^{11,12}$ In this work, we present evidence that this continues to hold for multicomponent fluids.

A major reason for the interest in the curvature dependence of surface tension is that it has a significant impact on the nucleation rates predicted by Classical Nucleation Theory since it affects the work of formation for a critical cluster. ${ }^{13,19-26}$ For pure water droplets nucleating in supersaturated vapor, incorporating the curvature dependence of the surface tension improves the agreement between the theory and the experimental results; ${ }^{27,28}$ the hope is that this also holds true for other substances and even for mixtures. Other applications of the Helfrich expansion include elastic properties of biological membranes, ${ }^{15,29}$ highly curved films,${ }^{16}$ and wetting at the nanoscale. ${ }^{30,31}$

Previous literature has dealt almost exclusively with one-component droplets; in reality, however, most interfaces contain several components. This paper deals with the curvature dependence of the surface tension for multicomponent fluids, which is conceptually more complicated. This is because for multicomponent droplets a variation in the radius can be achieved along several paths in thermodynamic variable space, e.g., by varying the pressure and composition of the exterior phase to different degrees. Since the surface tension will have a different dependence on the radius for different paths, the Tolman length and rigidity constants also acquire a pathdependence. This stands in stark contrast to the one-component isothermal case. The fact that thermodynamic properties can have a path dependence is a well-known concept; for instance, the heat capacity will differ depending on whether the temperature is varied at constant volume or at constant pressure.

Already in 1950, Koenig ${ }^{32}$ generalized Tolman's work to multicomponent systems, deriving analogous results for arbitrary directions through the thermodynamic variable space; however, the scope was restricted to first-order derivatives of the surface tension. A more thorough theoretical treatment was given by Groenewold and Bedeaux ${ }^{33}$ in 1995. They extended Koenig's work to incorporate second-order effects and, in the context of a statistical-mechanical treatment with pairwise additive potentials, presented the general equations for curvature effects on most thermodynamic properties of interest, including the surface tension.

The first to present quantitative estimates for the Tolman length of multicomponent droplets were Santiso and Firoozabadi. ${ }^{34}$ Apparently unaware of the studies by Koenig, ${ }^{32}$ and Groenewold and Bedeaux, ${ }^{33}$ a subset of earlier results was rediscovered for derivatives of the surface tension taken at constant composition of the exterior phase. The model employed by Santiso and Firoozabadi is a multicomponent extension ${ }^{35}$ of the Macleod ${ }^{36}$ correlation of surface tension in terms of bulk phase densities. By assuming that the Macleod equation applies also to curved surfaces, the resulting curvature dependence of the surface tension can be calculated solely from bulk phase properties. A general shortcoming of bulkphase correlations is that they are incapable of accurately representing surface enrichment and other surface-specific phenomena.

In this work, we give a general derivation of the theory for curvature-dependence of thermodynamic properties along arbitrary paths in thermodynamic variable space. In addition, a framework for computing Tolman lengths and rigidity constants of multicomponent fluids is presented. Instead of using bulk-property correlations, we use a more sophisticated DFT approach of minimizing the free energy as a functional of the density profiles. The theoretical foundation presented in this work is model-independent, but for the computations we shall particularize to Square Gradient Theory (SGT), which is a semi-empirical density functional theory that often captures the underlying physics of the droplet almost as well as more rigorous DFTs. ${ }^{37}$ In particular, SGT and a more sophisticated, non-local DFT have been shown to give very similar values for the Tolman length and rigidity constants of simple fluids. ${ }^{11}$

The structure of the paper is as follows. In Sec. II, we extend previous work on curvature corrections, beginning with the general and model-independent theory in Secs. II A and II B, and then present a framework for computing multicomponent Tolman lengths and rigidity constants by use of SGT. In Sec. III, we describe a numerical method for solving the resulting equations. In Sec. IV, we evaluate curvature corrections for the hexane-heptane mixture and describe some scenarios where curvature corrections for multicomponent droplets can be used. Finally, we offer some concluding remarks in Sec. V.

\section{THEORY}

For the equilibrium curvature of a droplet to change, there must be a change in the intensive thermodynamic variables of the system, i.e., the temperature or chemical potentials. Curvature corrections have previously been considered at isothermal conditions, ${ }^{2,5,6}$ and for the sake of simplicity, we shall also restrict ourselves to this situation. Letting $\boldsymbol{\mu}=\left(\mu_{1}, \ldots, \mu_{n}\right)$ be the vector of the chemical potentials of components 1 to $n$ and $\rho=\left(\rho_{1}, \ldots, \rho_{n}\right)$ be the corresponding densities, the Gibbs equation for the internal energy density can be written as $\mathrm{d} u=\boldsymbol{\mu} \mathrm{d} \rho$. Boldfaced symbols will represent vectors or matrices, and the inner product is implied when two vector quantities are written next to each other, i.e., $\boldsymbol{\mu} \mathrm{d} \rho=\boldsymbol{\mu} \cdot \mathrm{d} \rho$. For a vector, removing the boldfont and subscripting with tot will mean a summation over components; for example, the total density is $\rho_{\text {tot }}=\sum_{i} \rho_{i}$.

Although we focus on curvature changes due to isothermal variation of the chemical potentials, the theory in Secs. II A and II B can effortlessly be extended to include also temperature variations. As explained in a previous study, ${ }^{33}$ this can be achieved by letting $\boldsymbol{\mu}=\left(\mu_{1}, \ldots, \mu_{n}, T\right)$ and $\boldsymbol{\rho}=\left(\rho_{1}, \ldots\right.$, $\left.\rho_{n}, s\right)$, where $s$ is the entropy density and $T$ is the temperature; all the derivations in Secs. II A and II B then remain valid.

\section{A. Definition of the problem}

Starting from a Vapor-Liquid Equilibrium (VLE) state (subscript 0 ) at $T_{0}$ and $\mu_{0}$, we proceed along a path $\mathcal{P}$ into the 
metastable region, where states are characterized by chemical potentials $\boldsymbol{\mu} \neq \boldsymbol{\mu}_{0}$. This is illustrated schematically in Fig. 1 for a binary mixture. Each state along a particular path $\mathcal{P}$ corresponds to a droplet or a bubble in equilibrium with its surrounding phase, where chemical potentials and temperature are spatially uniform. We refer to previous studies for a discussion of thermodynamic stability, which depends on the boundary conditions. ${ }^{38-40}$ Even though droplets and bubbles are in equilibrium with a surrounding supersaturated phase, the term VLE state will in the following denote a saturation state; this entails that the vapor-liquid interface is flat.

Another route to arrive at expressions for the curvature coefficients is by considering the surface curvature associated with fluctuations of an otherwise planar surface $\left(\boldsymbol{\mu}=\boldsymbol{\mu}_{0}\right)$. The planar surface tension and curvature coefficients are then derived from considering the free energy of such surface waves at different lengthscales. This route, which was previously investigated for one-component systems, ${ }^{41-43}$ will not be further investigated here and we consider the VLE state only.

A spherical droplet or bubble is modeled as illustrated in Fig. 2, and we may define its radius $R$ corresponding to a choice of dividing surface. The droplet is contained in a spherical volume of radius $R_{\text {tot }}$, chosen so large that the surrounding phase has bulk behavior at the boundary. The radius can, for example, be chosen to correspond to the total equimolar radius $R_{\mathrm{e}}$, defined implicitly by

$$
N_{\mathrm{tot}}=V\left(R_{\mathrm{e}}\right) \rho_{\mathrm{tot}}(0)+\left(V\left(R_{\mathrm{tot}}\right)-V\left(R_{\mathrm{e}}\right)\right) \rho_{\mathrm{tot}}\left(R_{\mathrm{tot}}\right) .
$$

Here $\rho_{\text {tot }}(r)$ is the total number density at radial position $r$ according to the model; $V(R)=4 \pi R^{3} / 3$ is the volume up to position $R$; and $N_{\text {tot }}$ is the total number of particles in the container. Cylindrical geometries can be treated in a similar way. The equimolar radius is a well-defined function of $\boldsymbol{\mu}$, and writing the state functions along a path $\mathcal{P}$ as a function of $R$, we introduce the functions $\boldsymbol{\mu}(R), \sigma(R), \boldsymbol{\rho}^{\text {liq }}, \boldsymbol{\rho}^{\mathrm{vap}}$,

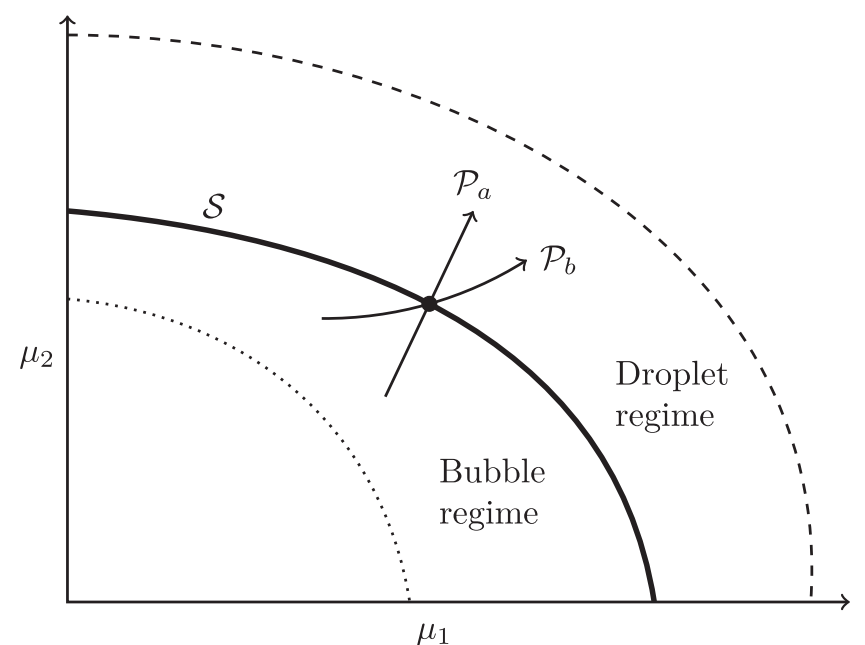

FIG. 1. Two possible paths in $\boldsymbol{\mu}$-space passing through a saturation state (black point), where the liquid surface curvature increases in the indicated direction. The shape of the phase envelope $\mathcal{S}$ is typical for VLE of two subcritical components. The dashed and dotted curves indicate the vapor and liquid spinodals, respectively.

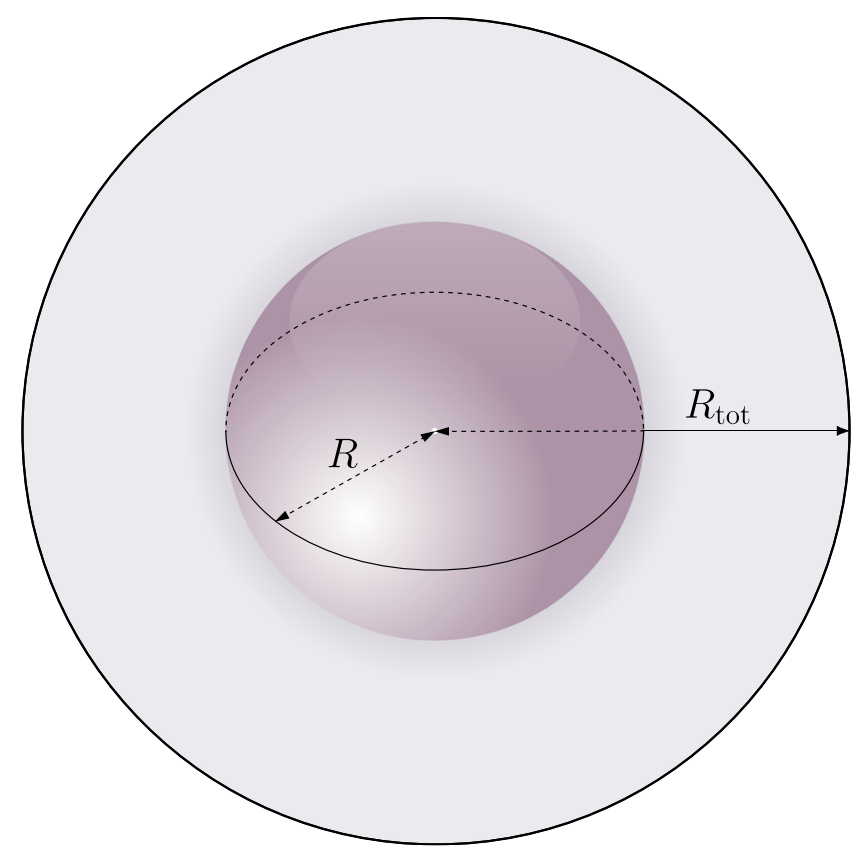

FIG. 2. A spherical droplet or bubble of radius $R$ inside a concentric spherical container of radius $R_{\text {tot }}$. The spherically symmetric density profiles $\rho(r)$ are approximately constant at the center $r=0$ and at the boundary $r=R_{\text {tot }}$, but generally have large gradients around the surface region $r=R$.

$P^{\text {liq }}$, etc., where superscripts liq and vap refer to the liquid and vapor phases, respectively. For a droplet, $\rho^{\text {liq }}=\rho(0)$ and $\rho^{\text {vap }}=\rho\left(R_{\text {tot }}\right)$. Note that properties like the curvaturedependent surface tension, $\sigma(R)$, are well-defined functions only when both the path $\mathcal{P}$ and the choice for the dividing surface are given. The phase envelope $\mathcal{S}$, consisting of the two-phase equilibrium states, is the $R=\infty$ isoradius contour corresponding to the flat surface.

\section{B. General relations from classical thermodynamics of surfaces}

We start by surveying the relevant relations from classical thermodynamics for surfaces. These are valid in all cases where the use of classical thermodynamics is justified, which, in particular, means that we assume the droplet/bubble to be large enough so that properties like $\rho^{\text {liq }}$ and $P^{\text {liq }}$ at the center have bulk phase behavior. These thermodynamic relations cannot give quantitative results before being coupled to a specific theoretical model, such as the SGT model introduced in Sec. II C.

\section{The Gibbs adsorption equation}

The surface tension is defined ${ }^{44}$ as $\sigma=\Omega^{\mathrm{E}} / A$, where $\Omega^{\mathrm{E}}$ is the excess (superscript $E$ ) grand free energy of a surface between two homogeneous bulk phases and $A$ is the surface area. From this definition, one can derive the Gibbs Adsorption Equation (GAE) ${ }^{45}$ For interface geometries where the curvature can be captured by a single variable $R$, it can be written as $^{44,46}$

$$
\mathrm{d} \sigma=-\boldsymbol{\Gamma} \mathrm{d} \boldsymbol{\mu}+\left[\frac{\partial \sigma}{\partial R}\right]_{T, \boldsymbol{\mu}} \mathrm{d} R .
$$

Here $R$ is usually the radius of a droplet or a bubble. The adsorption vector, $\boldsymbol{\Gamma}$, contains the excess number densities per 
area, while $[\partial \sigma / \partial R]_{T, \boldsymbol{\mu}}$ is the so-called notional derivative ${ }^{44,47}$ of the surface tension with respect to radius, i.e., the derivative of the surface tension with respect to the choice of radius when keeping the physical system ( $T$ and $\boldsymbol{\mu}$ ) unchanged. In addition to the notional derivative, one has the physical derivative of the surface tension. The physical derivative is well-defined only after having made a choice of dividing surface (such as the total equimolar radius), and a choice of path $\mathcal{P}$ through the thermodynamic variable space. For one-component droplets at fixed temperature, however, only a choice of dividing surface is needed since the only possible path through variable space that corresponds to droplets follows an increase in the pressure of the external phase. The chemical potential is then determined by the Gibbs-Duhem relation. The physical and notional derivatives of the surface tension are related by ${ }^{46,48}$

$$
\left[\frac{\partial \sigma}{\partial R}\right]_{T, \boldsymbol{\mu}}=\frac{\mathrm{d} \sigma}{\mathrm{d} R}+\boldsymbol{\Gamma} \frac{\mathrm{d} \boldsymbol{\mu}}{\mathrm{d} R},
$$

which follows directly from Eq. (5) by taking the physical derivative. Since $[\partial \sigma / \partial R]_{T, \boldsymbol{\mu}}$ is, in general, different from zero, it follows that for curved surfaces the surface tension depends on the choice of dividing surface.

Another expression for the notional derivative of the surface tension can be obtained from the fundamental property that a notional change in the radius must leave the physical system unchanged. It follows that the notional derivative of the grand canonical potential $[\partial \Omega / \partial R]_{T, \mu}$ is zero, from which one can deduce the generalized Laplace relation ${ }^{46-48}$

$$
\left[\frac{\partial \sigma}{\partial R}\right]_{T, \boldsymbol{\mu}}=\Delta P-\frac{g \sigma(R)}{R},
$$

where the geometry factor $g$ equals 1 for a cylindrical bubble/droplet, 2 for a spherical bubble/droplet and $\Delta P=P^{\text {liq }}$ $-P^{\mathrm{vap}}$, where the two pressures are taken in the homogeneous bulk phases. By use of the geometry factor $g$, our derivations below will be valid for both spherical and cylindrical geometries.

In addition to the total equimolar radius defined in Eq. (4), another popular choice of dividing surface is the surface of tension, defined by $[\partial \sigma / \partial R]_{T, \boldsymbol{\mu}}=0$. This is equivalent to the definition $\left[\partial \sigma / \partial\left(R^{-1}\right)\right]_{T, \boldsymbol{\mu}}=0$, which is more convenient for our purposes. As we will see, the theory becomes especially simple for this choice of dividing surface.

\section{Curvature expansions for a multicomponent fluid}

For a given choice of path $\mathcal{P}$ passing through a VLE state, and for any well-defined dividing surface, we can define the general curvature expansions as

$$
\begin{aligned}
\mu(R) & =\boldsymbol{\mu}_{0}+\frac{\boldsymbol{\mu}_{1}}{R}+\frac{\boldsymbol{\mu}_{2}}{R^{2}}+\cdots \\
\boldsymbol{\rho}(r ; R) & =\boldsymbol{\rho}_{0}(r)+\frac{\boldsymbol{\rho}_{1}(r)}{R}+\frac{\boldsymbol{\rho}_{2}(r)}{R^{2}}+\cdots, \\
\Delta P(R) & =0+\frac{\Delta P_{1}}{R}+\frac{\Delta P_{2}}{R^{2}}+\cdots, \\
\sigma(R) & =\sigma_{0}+\frac{\sigma_{1}}{R}+\frac{\sigma_{2}}{R^{2}}+\cdots, \\
\Gamma(R) & =\boldsymbol{\Gamma}_{0}+\frac{\boldsymbol{\Gamma}_{1}}{R}+\frac{\boldsymbol{\Gamma}_{2}}{R^{2}}+\cdots
\end{aligned}
$$

We emphasize that these expansions consider physical changes of the radius $R$, for a fixed definition of the dividing surface. Here $\rho(r ; R)$ is the density profile as a function of the radial coordinate $r$. The quantities we have introduced on the righthand sides are coefficients in an expansion with respect to $1 / R$, and thus these coefficients are independent of the value of $R$ for a given choice of dividing surface. As explained in Sec. I, $R>0$ for droplets and $R<0$ for bubbles in the curvature expansions, and thus $\boldsymbol{\mu}_{1}$ is directed into the droplet regime (cf. Fig. 1). Equations (8)-(12) are valid for both spherical and cylindrical geometries, with different values of the coefficients. The zero-subscripted quantities are properties of the VLE state, corresponding to a flat interface. The first- and second-order coefficients are subscripted with 1 and 2 and depend on the path $\mathcal{P}$ in thermodynamic variable space along which $R$ is varied. Note that the vector quantity $\boldsymbol{\mu}_{1}$ is different from the scalar quantity $\mu_{1}$, the latter being the chemical potential of component 1 .

The aim of this section is to derive general relations between the coefficients on the right-hand sides of Eqs. (8)-(12). Combining Eqs. (6) and (7), we find that $\Delta P=g \sigma(R) / R+\mathrm{d} \sigma(R) / \mathrm{d} R+\boldsymbol{\Gamma} \mathrm{d} \boldsymbol{\mu}(R) / \mathrm{d} R$, and expanding in $1 / R$ yields

$$
\begin{aligned}
\Delta P= & \frac{g \sigma_{0}}{R}+\frac{(g-1) \sigma_{1}-\boldsymbol{\mu}_{1} \boldsymbol{\Gamma}_{0}}{R^{2}} \\
& +\frac{(g-2) \sigma_{2}-\boldsymbol{\mu}_{1} \boldsymbol{\Gamma}_{1}-2 \boldsymbol{\mu}_{2} \boldsymbol{\Gamma}_{0}}{R^{3}} \cdots .
\end{aligned}
$$

By applying the Gibbs-Duhem equation, $\mathrm{d} P=\boldsymbol{\rho} \mathrm{d} \boldsymbol{\mu}$, separately to each of the two homogeneous phases, we find that $\mathrm{d} P^{\mathrm{liq}} / \mathrm{d} R=\rho^{\mathrm{liq}} \mathrm{d} \mu / \mathrm{d} R$ and $\mathrm{d} P^{\text {vap }} / \mathrm{d} R=\rho^{\text {vap }} \mathrm{d} \mu / \mathrm{d} R$, which upon subtraction becomes $\mathrm{d} \Delta P / \mathrm{d} R=\Delta \rho \mathrm{d} \mu / \mathrm{d} R$. By expanding this last equation in $1 / R$ and comparing the coefficients with Eq. (13), we obtain the relations

$$
\begin{aligned}
g \sigma_{0} & =\boldsymbol{\mu}_{1} \Delta \boldsymbol{\rho}_{0}, \\
(g-1) \sigma_{1}-\boldsymbol{\mu}_{1} \boldsymbol{\Gamma}_{0} & =\boldsymbol{\mu}_{2} \Delta \boldsymbol{\rho}_{0}+\frac{1}{2} \boldsymbol{\mu}_{1} \Delta \boldsymbol{\rho}_{1},
\end{aligned}
$$

where $\Delta \boldsymbol{\rho}_{1}=\boldsymbol{\rho}_{1}^{\text {liq }}-\boldsymbol{\rho}_{1}^{\mathrm{vap}}$. The directions of $\boldsymbol{\mu}_{1}$ and $\boldsymbol{\mu}_{2}$ vary according to the choice of dividing surface and path $\mathcal{P}$ considered in the thermodynamic variable space; however, their inner product with $\Delta \rho_{0}$ is fixed by Eqs. (14) and (15). In other words, one is free to choose the directions of $\boldsymbol{\mu}_{1}$ and $\boldsymbol{\mu}_{2}$, but not their norms. Referring to Fig. 1, the path $\mathcal{P}_{a}$ is a straight (i.e., linear) path in $\boldsymbol{\mu}$-space, which implies that $\boldsymbol{\mu}_{1} \propto \boldsymbol{\mu}_{2} \propto$ $\Delta \boldsymbol{\mu}$, where $\Delta \boldsymbol{\mu}=\boldsymbol{\mu}-\boldsymbol{\mu}_{0}$; the path $\mathcal{P}_{b}$ is curved and $\boldsymbol{\mu}_{1}$ and $\boldsymbol{\mu}_{2}$ have different directions. These vectors are not fully determined before one also knows their norms, and Eqs. (14) and (15) determine these last degrees of freedom.

The term $\Delta \rho_{1}$ in Eq. (15) can be re-expressed using properties of the planar density profile, by using that $\boldsymbol{\rho}_{1}^{\text {liq }}=\left(\boldsymbol{\rho}_{0 \boldsymbol{\mu}}^{\mathrm{liq}}\right) \boldsymbol{\mu}_{1}$, where $\boldsymbol{\rho}_{0 \boldsymbol{\mu}}^{\mathrm{liq}}$ is the derivative of $\boldsymbol{\rho}^{\mathrm{liq}}$ as a function ${ }^{49}$ of $\boldsymbol{\mu}$, evaluated at $\boldsymbol{\mu}_{0}$. Similarly, we have that $\rho_{1}^{\mathrm{vap}}=\left(\boldsymbol{\rho}_{0 \boldsymbol{\mu}}^{\mathrm{vap}}\right) \boldsymbol{\mu}_{1}$, and so

$$
\mu_{1} \Delta \rho_{1}=\mu_{1}^{\top}\left(\Delta \rho_{0 \mu}\right) \mu_{1},
$$

where $\Delta \boldsymbol{\rho}_{0 \boldsymbol{\mu}}=\boldsymbol{\rho}_{0 \boldsymbol{\mu}}^{\text {liq }}-\boldsymbol{\rho}_{0 \boldsymbol{\mu}}^{\mathrm{vap}}$. In Table I, we show how Eqs. (14) and (15) particularize for spherical and cylindrical geometries, using both the surface of tension and an arbitrary choice 
TABLE I. General relations between curvature-correction coefficients for cylindrical and spherical geometries. Results are shown for an arbitrary choice of dividing surface and the surface of tension. Quantities corresponding to a cylindrical or a spherical curvature are superscripted with, respectively, $\mathrm{c}$ or $\mathrm{s}$. These relations extend previous findings for the one-component case. ${ }^{41,50}$

\begin{tabular}{|c|c|c|}
\hline & Arbitrary dividing surface & Surface of tension \\
\hline \multirow{2}{*}{ Cylinder } & $\sigma_{0}=\boldsymbol{\mu}_{1}^{\mathrm{c}} \Delta \boldsymbol{\rho}_{0}$ & $\sigma_{0}=\boldsymbol{\mu}_{1}^{\mathrm{c}} \Delta \boldsymbol{\rho}_{0}$ \\
\hline & $0=\boldsymbol{\Gamma}_{0} \boldsymbol{\mu}_{1}^{\mathrm{c}}+\boldsymbol{\mu}_{2}^{\mathrm{c}} \Delta \boldsymbol{\rho}_{0}+\frac{1}{2}\left(\boldsymbol{\mu}_{1}^{\mathrm{c}}\right)^{\top}\left(\Delta \boldsymbol{\rho}_{0 \boldsymbol{\mu}}\right) \boldsymbol{\mu}_{1}^{\mathrm{c}}$ & $\sigma_{0} \delta=\boldsymbol{\Gamma}_{0} \boldsymbol{\mu}_{1}^{\mathrm{c}}$ \\
\hline \multirow{2}{*}{ Sphere } & $2 \sigma_{0}=\boldsymbol{\mu}_{1}^{\mathrm{s}} \Delta \boldsymbol{\rho}_{0}$ & $2 \sigma_{0}=\boldsymbol{\mu}_{1}^{\mathrm{s}} \Delta \boldsymbol{\rho}_{0}$ \\
\hline & $-2 \sigma_{0} \delta=\boldsymbol{\Gamma}_{0} \boldsymbol{\mu}_{1}^{\mathrm{s}}+\boldsymbol{\mu}_{2}^{\mathrm{s}} \Delta \boldsymbol{\rho}_{0}+\frac{1}{2}\left(\boldsymbol{\mu}_{1}^{\mathrm{s}}\right)^{\top}\left(\Delta \boldsymbol{\rho}_{0 \boldsymbol{\mu}}\right) \boldsymbol{\mu}_{1}^{\mathrm{s}}$ & $2 \sigma_{0} \delta=\boldsymbol{\Gamma}_{0} \boldsymbol{\mu}_{1}^{\mathrm{s}}$ \\
\hline
\end{tabular}

of dividing surface. The equations in Table I have been formulated in terms of the Tolman length, using the relation $\sigma_{1}=-g \sigma_{0} \delta$. Furthermore, Eq. (14) implies that $\Delta \boldsymbol{\mu}_{1}^{\mathrm{c}}=\frac{1}{2} \Delta \boldsymbol{\mu}_{1}^{\mathrm{s}}$; such a simple relationship does not in general exist between $\boldsymbol{\mu}_{2}^{\mathrm{c}}$ and $\boldsymbol{\mu}_{2}^{\mathrm{s}}$.

Equation (14) is not valid for paths that are tangential to the VLE envelope in $\mu$-space since we then have that $\boldsymbol{\mu}_{1} \Delta \boldsymbol{\rho}_{0}=0$, while $g \sigma_{0} \neq 0$. The reason for this is that the curvature is constant (zero) along the VLE envelope, which means that thermodynamic properties cannot be parametrized with the curvature. Each point on a valid path should correspond to a different value of the curvature. We shall discuss this point further in Secs. IV B and IV C.

We will also need formulae for the curvature expansion coefficients for the adsorption, $\boldsymbol{\Gamma}_{0}$ and $\boldsymbol{\Gamma}_{1}$. By definition, we have that $\boldsymbol{\Gamma}(R)=\int \boldsymbol{\rho}^{\mathrm{E}} \mathrm{d} \boldsymbol{r} / A(R)$, where $A(R)$ is the area of the cylindrical or spherical droplet/bubble. Using the geometry factor $g$, this can be written compactly as $\boldsymbol{\Gamma}(R)=\int \boldsymbol{\rho}^{\mathrm{E}}(z)(1+z / R)^{g} \mathrm{~d} z$. Here we have made the substitution $z=r-R$, which is conventional when dealing with curvature corrections ${ }^{50}$ A Taylor expansion in $1 / R$ then yields that

$$
\begin{aligned}
& \boldsymbol{\Gamma}_{0}=\int \boldsymbol{\rho}_{0}^{\mathrm{E}}(z) \mathrm{d} z, \\
& \boldsymbol{\Gamma}_{1}=\int\left(\boldsymbol{\rho}_{1}^{\mathrm{E}}(z)+g z \boldsymbol{\rho}_{0}^{\mathrm{E}}\right) \mathrm{d} z,
\end{aligned}
$$

where, in general, $\boldsymbol{\Gamma}_{1} \neq \int \rho_{1}^{\mathrm{E}}(z) \mathrm{d} z$.

\section{Role of the dividing surface}

For an arbitrary but fixed path $\mathcal{P}$ through the thermodynamic variable space, which is not parallel to the saturation locus, we now examine how the first- and second-order coefficients in Eqs. (8)-(12) depend on the choice of dividing surface, i.e., the definition of $R$. Consider two different definitions of the dividing surface, according to which the radius of a given droplet is, respectively, $\widetilde{R}$ and $R$. Following previous notation, ${ }^{46}$ we assume that we can write

$$
\begin{aligned}
\widetilde{R} & =R+\epsilon(R) \\
& =R+\epsilon_{0}+\frac{\epsilon_{1}}{R}+\cdots .
\end{aligned}
$$

This assumption is equivalent to the requirement that the deviation $\widetilde{R}-R$ is finite in the planar limit, and that it varies smoothly with the curvature, $1 / R$. This can also be formulated as

$$
\frac{1}{\widetilde{R}}=\frac{1}{R}-\frac{\epsilon_{0}}{R^{2}}+\frac{\epsilon_{0}^{2}-\epsilon_{1}}{R^{3}}+\cdots,
$$

which is a more convenient form when evaluating curvature expansions.

Clearly, a different definition of the dividing surface will not change the physical properties of the system, and in particular, it will not change the chemical potential $\mu$. This means that we can write $\boldsymbol{\mu}(R)=\widetilde{\boldsymbol{\mu}}(\widetilde{R})$, where the left- and right-hand sides denote the chemical potentials as functions of $R$ and $\widetilde{R}$, respectively, as these vary along $\mathcal{P}$. By Taylor expanding the right-hand side of this identity in $1 / \widetilde{R}$ and using Eq. (20) to replace $1 / \widetilde{R}$ with $1 / R$, we find that

$$
\begin{aligned}
& \widetilde{\boldsymbol{\mu}}_{0}=\boldsymbol{\mu}_{0}, \\
& \widetilde{\boldsymbol{\mu}}_{1}=\boldsymbol{\mu}_{1}, \\
& \widetilde{\boldsymbol{\mu}}_{2}=\boldsymbol{\mu}_{2}+\epsilon_{0} \boldsymbol{\mu}_{1} .
\end{aligned}
$$

Similarly, we have that $\Delta P(R)=\widetilde{\Delta P}(\widetilde{R})$ so that

$$
\begin{aligned}
& \widetilde{\Delta P_{0}}=\Delta P_{0}, \\
& \widetilde{\Delta P_{1}}=\Delta P_{1}, \\
& \widetilde{\Delta P_{2}}=\Delta P_{2}+\epsilon_{0} \Delta P_{1} .
\end{aligned}
$$

Indeed, for any quantity $h$ that is independent of the dividing surface, we have that $\widetilde{h}_{0}=h_{0}, \widetilde{h}_{1}=h_{1}$, and $\widetilde{h}_{2}=h_{2}+\epsilon_{0} h_{1}$. Equation (23) shows that the direction of $\boldsymbol{\mu}_{2}$ is not fully determined by the path $\mathcal{P}$, but in general also depends on the choice of dividing surface.

Regarding the density profile, a curvature expansion of the identity $\rho(z+\epsilon(R))=\widetilde{\rho}(z)$ yields that

$$
\begin{aligned}
& \widetilde{\boldsymbol{\rho}}_{0}(z)=\boldsymbol{\rho}_{0}\left(z+\epsilon_{0}\right), \\
& \widetilde{\boldsymbol{\rho}}_{1}(z)=\boldsymbol{\rho}_{1}\left(z+\epsilon_{0}\right)+\epsilon_{1} \boldsymbol{\rho}_{0 z}\left(z+\epsilon_{0}\right),
\end{aligned}
$$

where $\boldsymbol{\rho}_{0 z}$ is the derivative of $\boldsymbol{\rho}_{0}$ with respect to $z$. It turns out that the quantity $\rho_{2}$ is not needed up to the order we consider in this framework ${ }^{50}$ and neither is $\boldsymbol{\Gamma}_{2}$. For the adsorptions, we insert Eqs. (27) and (28) into Eqs. (17) and (18) and obtain

$$
\begin{aligned}
& \widetilde{\boldsymbol{\Gamma}}_{0}=\boldsymbol{\Gamma}_{0}-\epsilon_{0} \Delta \boldsymbol{\rho}_{0}, \\
& \widetilde{\boldsymbol{\Gamma}}_{1}=\boldsymbol{\Gamma}_{1}-\epsilon_{0}\left(\Delta \boldsymbol{\rho}_{1}+g \boldsymbol{\Gamma}_{0}\right)-\epsilon_{1} \Delta \boldsymbol{\rho}_{0}+\frac{g}{2} \epsilon_{0}^{2} \Delta \boldsymbol{\rho}_{0} .
\end{aligned}
$$

Especially interesting are the curvature expansion coefficients for the surface tension,

$$
\begin{aligned}
& \widetilde{\sigma}_{0}=\sigma_{0}, \\
& \widetilde{\sigma}_{1}=\sigma_{1}, \\
& \widetilde{\sigma}_{2}=\sigma_{2}-\epsilon_{0} \boldsymbol{\mu}_{1} \boldsymbol{\Gamma}_{0}+\frac{g}{2} \epsilon_{0}^{2} \sigma_{0} .
\end{aligned}
$$

In terms of the Helfrich coefficients, Eqs. (32) and (33) lead to

$$
\begin{aligned}
\widetilde{\delta} & =\delta, \\
\widetilde{k} & =k-2 \epsilon_{0} \boldsymbol{\mu}_{1}^{\mathrm{c}} \boldsymbol{\Gamma}_{0}+\epsilon_{0}^{2} \sigma_{0}, \\
\widetilde{(2 k+\bar{k})} & =2 k+\bar{k}-\epsilon_{0} \boldsymbol{\mu}_{1}^{\mathrm{s}} \boldsymbol{\Gamma}_{0}+\epsilon_{0}^{2} \sigma_{0} .
\end{aligned}
$$

Equation (34) shows that the Tolman length is independent of the choice of dividing surface also for multicomponent fluids, which extends previous findings for a one-component system. ${ }^{11}$ Equations (35) and (36) are simple transformation 
formulae for the rigidity constants of multicomponent fluids for different choices of dividing surface. To derive the transformation formulae for the surface tension, we use the definition of the notional derivative to write

$$
\sigma(\widetilde{R})=\sigma(R)+\int_{R}^{\widetilde{R}}\left[\frac{\mathrm{d} \sigma}{\mathrm{d} R^{\prime}}\right]_{T, \mu} \mathrm{d} R^{\prime} .
$$

Taylor-expand the right-hand side to second order in $1 / \widetilde{R}$, and compare coefficients with the second-order expansion of $\widetilde{\sigma}(\widetilde{R})$. This is a lengthy, but straightforward derivation that can be found in the supplementary material.

All the transformation formulae Eqs. (21)-(36) have been verified numerically using the SGT model, in the specific case where $\widetilde{R}$ is the total equimolar radius and $R$ is the radius of tension.

For the one-component case, it was shown in a previous study ${ }^{11}$ that for a particular DFT model, the equimolar surface is the choice of dividing surface that minimizes the magnitude of the rigidity constants. Equations (34)-(36) constitute a generalization of this result to a multicomponent fluid, and the derivation shows that it is not a peculiarity of the DFT model in Ref. 11, but generally true for one-component fluids. In the multicomponent case, any dividing surface that satisfies $\boldsymbol{\mu}_{1} \boldsymbol{\Gamma}_{0}=0$ will minimize $\sigma_{2}$. One example of such a radius is the $(\mathrm{d} \mu / \mathrm{d} R)$-weighted equimolar radius defined by $\Gamma(\mathrm{d} \mu / \mathrm{d} R)=0$, and another is the one defined by $\boldsymbol{\mu}_{1} \boldsymbol{\Gamma}=0$. Another property that these choices of radii have in common is that a multicomponent version of Tolman's law holds. ${ }^{32,46}$ The main drawback of any definition for the choice of dividing surface that satisfies $\boldsymbol{\mu}_{1} \boldsymbol{\Gamma}_{0}=0$ for a multicomponent fluid, is that it will not be a state function since it depends on the direction of $\boldsymbol{\mu}_{1}$; thus the same physical droplet will be assigned a different radius according to which $\boldsymbol{\mu}_{1}$ that is considered.

\section{Computing the curvature coefficients by use of SGT}

Curvature corrections for the SGT model have been derived in the literature for one-component fluids. ${ }^{50}$ In the following, we derive similar corrections for a multicomponent fluid interface as described by SGT. The multicomponent expressions will have the same form as in the singlecomponent case, to which they reduce in the one-dimensional case. In SGT, the grand free energy functional is modeled as

$$
\Omega[\boldsymbol{\rho}]=\int\left(\frac{1}{2} \boldsymbol{\rho}_{r}^{\top} \boldsymbol{K} \boldsymbol{\rho}_{r}+a^{\operatorname{EOS}}(\boldsymbol{\rho})-\boldsymbol{\mu} \rho\right) \mathrm{d} \boldsymbol{r},
$$

where subscript $r$ means a derivative with respect to $r$. Here $\boldsymbol{K}$ is a constant, symmetric and positive semidefinite $n \times n$ matrix known as the influence matrix, and $a^{\mathrm{EOS}}(\rho)$ is the Helmholtz energy density of a fluid with uniform densities $\rho$ at the specified temperature. The function $a^{\mathrm{EOS}}$ is modeled with an Equation of State (EOS), where $a^{\mathrm{EOS}}(\rho)=-P^{\mathrm{EOS}}(\rho)$ $+\mu^{\mathrm{EOS}}(\rho) \rho$. Since we restrict the discussion to planar, cylindrical, and spherical geometries, only one spatial variable $r$ is needed. The Laplacian can then be written as $\nabla^{2}=\mathrm{d}^{2} / \mathrm{d} r^{2}+(g / r) \mathrm{d} / \mathrm{d} r$, where $g$ is 0,1 , or 2 depending on the geometry. The stationarity condition $\delta \Omega[\rho]=0$ is found to be equivalent to the system of differential equations $-\boldsymbol{K} \nabla^{2} \rho+\mu^{\mathrm{EOS}}(\rho)-\boldsymbol{\mu}=\mathbf{0}$, or

$$
\boldsymbol{K} \boldsymbol{\rho}_{r r}=-\frac{g}{r} \boldsymbol{K} \boldsymbol{\rho}_{r}+\boldsymbol{\mu}^{\mathrm{EOS}}(\boldsymbol{\rho})-\boldsymbol{\mu},
$$

where subscript $r r$ means the second-order derivative with respect to $r$. Similar to the one-component case, ${ }^{50}$ we can Taylor-expand Eq. (38) in $1 / R$, and by using the definitions Eqs. (8) and (9), we find that

$$
\begin{aligned}
& \boldsymbol{K} \boldsymbol{\rho}_{0 z z}=\boldsymbol{\mu}^{\mathrm{EOS}}\left(\boldsymbol{\rho}_{0}\right)-\boldsymbol{\mu}_{0}, \\
& \boldsymbol{K} \boldsymbol{\rho}_{1 z z}=\boldsymbol{\mu}_{\boldsymbol{\rho}}^{\mathrm{EOS}}\left(\boldsymbol{\rho}_{0}\right) \boldsymbol{\rho}_{1}-\boldsymbol{\mu}_{1}-g \boldsymbol{K} \boldsymbol{\rho}_{0 z},
\end{aligned}
$$

where $z=R-r$ as in Sec. II B 3 and subscripts $z$ and $z z$ mean first- and second-order derivatives with respect to $z$. Since $\boldsymbol{\mu}_{1}^{\mathrm{c}}=\frac{1}{2} \boldsymbol{\mu}_{1}^{\mathrm{s}}$, we have that $\boldsymbol{\rho}_{1}^{\mathrm{c}}=\frac{1}{2} \boldsymbol{\rho}_{1}^{\mathrm{s}}$ for SGT.

The solutions of Eq. (40) are degenerate: if $\rho_{1}$ is a solution, then $\boldsymbol{\rho}_{1}+\alpha \boldsymbol{\rho}_{0 z}$ is as well. This reflects the fact that the firstorder curvature correction for the density, $\rho_{1}$, depends on the choice of dividing surface, where we refer to Eq. (28) and the discussion in Sec. II B 3 for more details. For the total equimolar surface, defined by $\Gamma_{\text {tot }}=0$, the constraint on $\rho_{1}$ is obtained from Eq. (18) by summing over the components

$$
\int\left[\rho_{1, \text { tot }}^{\mathrm{E}}(z)+g z \rho_{0, \mathrm{tot}}^{\mathrm{E}}(z)\right] \mathrm{d} z=0 .
$$

Equation (41) restores uniqueness for the total equimolar surface and has been stated before in the literature for the onecomponent case.$^{50}$ For the surface of tension, it follows from the Appendix that the condition on $\rho_{1}$ is

$$
\int \boldsymbol{\rho}_{0 z}^{\top} \boldsymbol{K} \boldsymbol{\rho}_{1} \mathrm{~d} z=\int\left[(g-1) z^{2} \boldsymbol{\rho}_{0 z}^{\top} \boldsymbol{K} \boldsymbol{\rho}_{0 z}+z^{2} \boldsymbol{\mu}_{1}^{\mathrm{s}} \boldsymbol{\rho}_{0 z}+z \boldsymbol{\mu}_{1}^{\mathrm{s}} \boldsymbol{\rho}_{0}^{\mathrm{E}}\right] \mathrm{d} z .
$$

For our purposes, there is no need to worry about the degeneracy of $\rho_{1}$, as the SGT formulae Eqs. (48)-(50) for the Helfrich coefficients are invariant under the shift $\rho_{1} \mapsto \rho_{1}+\alpha \rho_{0 z}$. For other applications, however, this degeneracy cannot be ignored, for example, when considering transport of heat and mass across curved vapor-liquid interfaces..$^{51,52}$

The surface tension equals the area-specific excess grand free energy $\Omega^{\mathrm{E}}[\rho] / A(R)$, which for SGT is given by

$$
\frac{\Omega^{\mathrm{E}}[\boldsymbol{\rho}]}{A(R)}=\int\left[\frac{1}{2} \boldsymbol{\rho}_{z}^{\top} \boldsymbol{K} \boldsymbol{\rho}_{z}+a^{\mathrm{E}}(\boldsymbol{\rho})-\boldsymbol{\mu} \boldsymbol{\rho}^{\mathrm{E}}\right]\left(1+\frac{z}{R}\right)^{g} \mathrm{~d} z,
$$

where $a^{\mathrm{E}}(\boldsymbol{\rho})=a^{\mathrm{EOS}}(\boldsymbol{\rho})-a^{\mathrm{EOS}}\left(\boldsymbol{\rho}^{\text {bulk }}\right)$ and, for a droplet, $\rho^{\text {bulk }}=H(-z) \rho^{\text {liq }}+H(z) \rho^{\text {vap }}$, where $H$ is the Heaviside function. The expression in Eq. (43) is valid for planar, cylindrical, and spherical geometries. Using the differential equations (39) and (40), one can evaluate the first few terms in a curvature expansion of Eq. (43). Details on this derivation are provided in the supplementary material. The final expression is

$$
\begin{aligned}
\frac{\Omega^{\mathrm{E}}[\boldsymbol{\rho}]}{A(R)}= & \int \boldsymbol{\rho}_{0 z}^{\top} \boldsymbol{K} \boldsymbol{\rho}_{0 z} \mathrm{~d} z \\
& +\frac{1}{R} \int\left[g z \boldsymbol{\rho}_{0 z}^{\top} \boldsymbol{K} \boldsymbol{\rho}_{0 z}-\boldsymbol{\mu}_{1}^{\top} \boldsymbol{\rho}_{0}^{\mathrm{E}}\right] \mathrm{d} z \\
& +\frac{1}{R^{2}} \int\left[\frac{g(g-1)}{2} z^{2} \boldsymbol{\rho}_{0 z}^{\top} \boldsymbol{K} \boldsymbol{\rho}_{0 z}-\frac{1}{2} g \boldsymbol{\rho}_{0 z}^{\top} \boldsymbol{K} \boldsymbol{\rho}_{1}\right. \\
& \left.+\frac{1}{2} g z^{2} \boldsymbol{\mu}_{1}^{\top} \boldsymbol{\rho}_{0 z}-\frac{1}{2} \boldsymbol{\mu}_{1}^{\top} \boldsymbol{\rho}_{1}^{\mathrm{E}}-\boldsymbol{\mu}_{2}^{\top} \boldsymbol{\rho}_{0}^{\mathrm{E}}\right] \mathrm{d} z .
\end{aligned}
$$


Comparing the curvature expansion in Eqs. (44)-(46) with the Helfrich expansion for the sphere [Eq. (2)] and cylinder [Eq. (3)], we find the following SGT expressions for the coefficients in the Helfrich expansion:

$$
\begin{aligned}
\sigma_{0}= & \int \boldsymbol{\rho}_{0 z}^{\top} \boldsymbol{K} \boldsymbol{\rho}_{0 z} \mathrm{~d} z \\
\delta= & -\frac{1}{\sigma_{0}} \int\left[z \boldsymbol{\rho}_{0 z}^{\top} \boldsymbol{K} \boldsymbol{\rho}_{0 z}+\frac{1}{2} \boldsymbol{\mu}_{1}^{\mathrm{s}} \boldsymbol{\rho}_{0}^{\mathrm{E}}\right] \mathrm{d} z \\
k= & \int\left[-\frac{1}{2} \boldsymbol{\rho}_{0 z}^{\top} \boldsymbol{K} \boldsymbol{\rho}_{1}^{\mathrm{s}}+\frac{1}{2} z^{2} \boldsymbol{\mu}_{1}^{\mathrm{s}} \boldsymbol{\rho}_{0 z}-\frac{1}{4} \boldsymbol{\mu}_{1}^{\mathrm{s}} \boldsymbol{\rho}_{1}^{\mathrm{s}, \mathrm{E}}-2 \boldsymbol{\mu}_{2}^{\mathrm{c}} \boldsymbol{\rho}_{0}^{\mathrm{E}}\right] \mathrm{d} z \\
\bar{k}= & \int\left[z^{2} \boldsymbol{\rho}_{0 z}^{\top} \boldsymbol{K} \boldsymbol{\rho}_{0 z}+\left(4 \boldsymbol{\mu}_{2}^{\mathrm{c}}-\boldsymbol{\mu}_{2}^{\mathrm{s}}\right) \boldsymbol{\rho}_{0}^{\mathrm{E}}\right] \mathrm{d} z \\
2 k+\bar{k}= & \int\left[z^{2} \boldsymbol{\rho}_{0 z}^{\top} \boldsymbol{K} \boldsymbol{\rho}_{0 z}-\boldsymbol{\rho}_{0 z}^{\top} \boldsymbol{K} \boldsymbol{\rho}_{1}^{\mathrm{s}}+z^{2} \boldsymbol{\mu}_{1}^{\mathrm{s}} \boldsymbol{\rho}_{0 z}-\frac{1}{2} \boldsymbol{\mu}_{1}^{\mathrm{s}} \boldsymbol{\rho}_{1}^{\mathrm{s}, \mathrm{E}}\right. \\
& \left.-\boldsymbol{\mu}_{2}^{\mathrm{s}} \boldsymbol{\rho}_{0}^{\mathrm{E}}\right] \mathrm{d} z
\end{aligned}
$$

Here, we have eliminated $\rho_{1}^{\mathrm{c}}$ and $\boldsymbol{\mu}_{1}^{\mathrm{c}}$ by using the relations $\rho_{1}^{\mathrm{c}}=\frac{1}{2} \rho_{1}^{\mathrm{s}}$ and $\boldsymbol{\mu}_{1}^{\mathrm{c}}=\frac{1}{2} \boldsymbol{\mu}_{1}^{\mathrm{s}}$. One can verify that the formulae in Eqs. (47)-(51) are consistent with the transformation formulae in Eqs. (31)-(33), both directly by analytical integration and numerically. This is an excellent consistency check for the SGT formulae since any consistent model must yield expressions for the Tolman lengths and rigidity constants that conform to these transformation formulae.

\section{NUMERICAL METHODS}

To determine the Tolman length and both rigidity constants along a path $\mathcal{P}$ in the thermodynamic variable space, we carried out the calculations in the following order:

(i) Solve the differential equation (39) for the planar density profile $\rho_{0}$ at the saturation state.

(ii) Obtain the direction of $\boldsymbol{\mu}_{1}^{\mathrm{s}}$ from the tangent of $\mathcal{P}$ in the $\boldsymbol{\mu}$-space. Use Eq. (14) to determine its magnitude.

(iii) Determine the Tolman length, $\delta$ from Eq. (48).

(iv) Solve the differential equation (40) for $\boldsymbol{\rho}_{1}^{\mathrm{s}}$, i.e., the first-order curvature correction to the density profile for spherical droplets.

(v) Determine the directions of $\boldsymbol{\mu}_{2}^{\mathrm{s}}$ and $\boldsymbol{\mu}_{2}^{\mathrm{c}}$ from $\mathcal{P}$, and determine their magnitudes using Eq. (15).

(vi) Determine the rigidity constants $k$ and $\bar{k}$ from Eqs. (49) and (50).

For a given path the first-order coefficients $\delta$ and $\boldsymbol{\mu}_{1}$ are independent of the choice of dividing surface, while the second-order coefficients $\boldsymbol{\mu}_{2}, k$, and $\bar{k}$ are not.

The most popular version of SGT is the so-called geometric mean square gradient theory (GM-SGT), where the combining rule for the influence matrix $\boldsymbol{K}=\left[\kappa_{i j}\right]_{i j}$ is given by the geometric mean of the pure component values, $\kappa_{i j}=\sqrt{\kappa_{i i} \kappa_{j j}}$. We will use GM-SGT to generate the results in Sec. IV, but in Secs. III A and III B below, we develop the numerical solution procedure for an arbitrary symmetric and positive semidefinite influence matrix $\boldsymbol{K}$.

\section{A. Solving the full SGT model with an arbitrary symmetric influence matrix}

We first discuss the numerical solution of the full SGT equation

$$
\boldsymbol{K} \nabla^{2} \rho(r)=\boldsymbol{\mu}^{\mathrm{EOS}}(\rho(r))-\boldsymbol{\mu} .
$$

Since $\boldsymbol{K}$ is symmetric we can write $\boldsymbol{K}=\boldsymbol{P} \boldsymbol{\Lambda} \boldsymbol{P}^{\top}$, where $\boldsymbol{P}$ is the orthogonal matrix having eigenvectors as columns and $\boldsymbol{\Lambda}$ is the diagonal matrix of eigenvalues. Let now $\iota$ be the tuple of indices corresponding to nonzero eigenvalues, and $\zeta$ be the tuple of indices of zero eigenvalues. Introducing the variables

$$
\boldsymbol{q}(r)=\boldsymbol{P}^{\top} \boldsymbol{\rho}(r), \quad \boldsymbol{\phi}(\boldsymbol{\rho}(r))=\boldsymbol{P}^{\top}\left(\boldsymbol{\mu}^{\mathrm{EOS}}(\rho(r))-\boldsymbol{\mu}\right),
$$

we reformulate Eq. (52) as a coupled set of differential equations and algebraic equations

$$
\begin{gathered}
\Lambda_{\iota} \nabla^{2} \boldsymbol{q}_{\iota}(r)=\phi_{\iota}(\rho(r)), \\
\boldsymbol{P}_{\iota}^{\top} \boldsymbol{\rho}(r)=\boldsymbol{q}_{\iota}(r), \quad \boldsymbol{\phi}_{\zeta}(\rho(r))=\mathbf{0},
\end{gathered}
$$

corresponding to rank $\boldsymbol{K}$ differential equations for $\boldsymbol{q}_{\iota}(r)$ and $n$ algebraic equations to recover $\boldsymbol{\rho}(r)$ given $\boldsymbol{q}_{\iota}(r)$. Of these algebraic equations, there are rank $\boldsymbol{K}$ linear equations and $n-\operatorname{rank} \boldsymbol{K}$ nonlinear equations that will have to be solved in each grid point. We use the Neumann boundary conditions

$$
\frac{\mathrm{d} \boldsymbol{q}_{\iota}}{\mathrm{d} r}(0)=\mathbf{0}, \quad \frac{\mathrm{d} \boldsymbol{q}_{\iota}}{\mathrm{d} r}\left(R_{\mathrm{tot}}\right)=\mathbf{0} .
$$

The first boundary condition always holds due to symmetry and is also necessary to ensure that the Laplacian does not diverge for the curved geometries. The second boundary condition is an approximation that becomes exact in the limit $R_{\text {tot }} \rightarrow \infty$; in practice, the densities approach their bulk values exponentially fast, akin to the exact tanh solution obtained when solving SGT using the van der Waals equation of state. ${ }^{44,53}$ One should verify that the spherical container is chosen sufficiently large so that the $\partial \boldsymbol{q}_{\iota} / \partial r$ vanishes well before the container wall at $r=R_{\text {tot }}$. Equations (53)-(55) constitute a nonlinear Boundary Value Problem (BVP).

In addition to Eqs. (53)-(55), which are true for any density profile, additional equations are needed to specify the particular solution we are interested in; this is equivalent to specifying $\boldsymbol{\mu}$ in Eq. (52). Instead of directly specifying the given $\mu$, it is equivalent and more convenient to specify a path in $\mu$-space as well as a value for the equimolar radius $R_{\mathrm{e}}$. As shown in previous work, ${ }^{40}$ this is most conveniently done by also solving for the vector of cumulative number of particles, $N(r)$, by use of the following equation:

$$
\frac{\mathrm{d} N(r)}{\mathrm{d} r}=A(r) \rho_{\mathrm{tot}}(r),
$$

and the boundary conditions $N(0)=0$ and Eq. (4), where $N\left(R_{\text {tot }}\right)=N_{\text {tot }}$ Restricting the considered paths to straight lines in $\boldsymbol{\mu}$-space, we have that $\boldsymbol{\mu}(t)=\boldsymbol{\mu}_{0}+t \boldsymbol{\mu}_{1}$, where $t$ is an unknown parameter that is part of the solution. A good initial guess for $t$ is $1 / R$.

As an initial guess for the density profiles, we use for each component a tanh function shifted and scaled so that it is centered at the position of the radius and has the bulk liquid and vapor densities as limiting values. We solve the algebraic equations defined by Eq. (54) with Newton's method. This 
entails that for each gridpoint we converge the iteration given by $\operatorname{DG}\left(\rho_{n}\right)\left(\rho_{n+1}-\rho_{n}\right)=\boldsymbol{G}\left(\boldsymbol{\rho}_{n}\right)$, where

$$
\boldsymbol{G}(\boldsymbol{\rho})=\left[\begin{array}{c}
\boldsymbol{P}_{\iota}^{\top} \boldsymbol{\rho}-\boldsymbol{q}_{\iota} \\
\boldsymbol{\phi}_{\zeta}(\boldsymbol{\rho})
\end{array}\right], \quad \mathrm{D} \boldsymbol{G}(\boldsymbol{\rho})=\left[\begin{array}{c}
\boldsymbol{P}_{\iota}^{\top} \\
\boldsymbol{P}_{\zeta}^{\top} \boldsymbol{\mu}_{\boldsymbol{\rho}}^{\mathrm{EOS}}(\rho)
\end{array}\right] .
$$

A good initial guess is available from the solution at the same position in the previous pass of the BVP solver, or the initial density profile if it is the solver's very first pass.

To solve the resulting BVP numerically, we use the collocation method solve_bvp in the Python library SciPy, ${ }^{54}$ version 0.18 .1 . The underlying algorithm is essentially the same algorithm as the widely used bvp4c integrator in MatLaв. One of its main features is automatic grid refinement, which in practice will produce a fine mesh in the interface region and a coarse mesh where the densities are essentially constant.

\section{B. Solving for the curvature corrections}

Using the solution $\rho_{0}$ for the planar density profile, we solve $\boldsymbol{K} \boldsymbol{\rho}_{1 z z}=\boldsymbol{\mu}_{\boldsymbol{\rho}}^{\mathrm{EOS}}\left(\boldsymbol{\rho}_{0}\right) \boldsymbol{\rho}_{1}-\boldsymbol{\mu}_{1}-g \boldsymbol{K} \boldsymbol{\rho}_{0 z}$ as a system of linear differential equations for $\rho_{1}$. Since solve_bvp uses automatic grid refinement, we use a cubic spline to interpolate $\rho_{0}$ in any new grid points the algorithm introduces. Defining

$$
\begin{aligned}
\boldsymbol{q}_{1}(z) & =\boldsymbol{P}^{\top} \boldsymbol{\rho}_{1}(z), \\
\boldsymbol{\phi}_{1}\left(\boldsymbol{\rho}_{1}(z)\right) & =\boldsymbol{P}^{\top}\left(\boldsymbol{\mu}_{\boldsymbol{\rho}}^{\mathrm{EOS}}\left(\boldsymbol{\rho}_{0}(z)\right) \boldsymbol{\rho}_{1}(z)-\boldsymbol{\mu}_{1}-g \boldsymbol{K} \boldsymbol{\rho}_{0 z}(z)\right),
\end{aligned}
$$

the equations to solve are

$$
\begin{gathered}
\boldsymbol{\Lambda}_{\iota} \boldsymbol{q}_{1 z z}(z)=\boldsymbol{\phi}_{1, \iota}\left(\boldsymbol{\rho}_{1}(z)\right), \\
\boldsymbol{P}_{\iota}^{\top} \boldsymbol{\rho}_{1}(z)=\boldsymbol{q}_{1, \iota}(z), \quad \boldsymbol{\phi}_{1, \zeta}\left(\boldsymbol{\rho}_{1}(z)\right)=\mathbf{0} .
\end{gathered}
$$

To solve for the curvature corrections is easier than finding a full SGT solution from Eqs. (53) and (54): solving for $\boldsymbol{q}_{1, \iota}$ from Eq. (58) amounts to solving a set of linear differential equations, in contrast to the nonlinear differential equations that determine $\boldsymbol{q}_{i}$; moreover, Eq. (59) is a linear system of algebraic equations, while Eq. (54) is nonlinear. We find that the initial guess $\boldsymbol{\rho}_{1}=\boldsymbol{0}$ suffices to converge the equations.

To calculate $\delta, k$, and $\bar{k}$, it suffices to solve Eqs. (58) and (59) for the spherical curvature-correction $\rho_{1}^{\mathrm{s}}$ since the cylindrical correction can be found from $\rho_{1}^{\mathrm{c}}=\frac{1}{2} \rho_{1}^{\mathrm{s}}$. To solve for $\rho_{1}$, we need the compositional Jacobian matrix of the chemical potentials $\boldsymbol{\mu}_{\boldsymbol{\rho}}^{\mathrm{EOS}}\left(\boldsymbol{\rho}_{0}\right)$. This Jacobian is usually available for the EOS, as it is needed for efficient thermodynamic algorithms such as flash algorithms.

For boundary conditions, we specify that the gradients vanish at the center of the droplet and at the container wall

$$
\frac{\mathrm{d} \boldsymbol{q}_{1, \iota}}{\mathrm{d} r}(0)=\mathbf{0}, \quad \frac{\mathrm{d} \boldsymbol{q}_{1, \iota}}{\mathrm{d} r}\left(R_{\mathrm{tot}}\right)=\mathbf{0},
$$

where the first condition again follows from a symmetry argument, while the latter is an approximation that becomes exact in the limit $R_{\mathrm{tot}} \rightarrow \infty$.

\section{Numerical consistency checks}

It is challenging to achieve a numerically robust implementation of SGT, and developing various approaches for this is an active research area. ${ }^{55-57}$ To ensure a correct implementation, the code has been subjected to the following consistency checks:

- The second-order expansion fits well with the surface tension values from the full SGT model for large droplets (see Fig. 10).

- The curvature correction transformation formulae in Sec. II B 3 hold in the case $\boldsymbol{\mu}_{1} \propto \boldsymbol{\mu}_{2}$ (i.e., straight path in $\boldsymbol{\mu}$-space) for the surface of tension vs. the total equimolar surface.

- The results remain unchanged when solving for a droplet of a given radius and in a larger container. This verifies that finite-size effects are negligible.

- The results remain unchanged when solving on a more refined grid, i.e., to a higher numerical accuracy.

- The Helfrich coefficients for the mixture approach their pure-component values as the mixture becomes very concentrated in one of the components.

\section{RESULTS AND DISCUSSION}

We discuss next the Tolman length, rigidity constants, and the accuracy of the Helfrich expansion, using the hexaneheptane mixture at $T=298.15 \mathrm{~K}$ as an example. We have checked that the conclusions drawn in this section are not sensitive to the choice of temperature by repeating the calculations at $T=330 \mathrm{~K}$ and $T=375 \mathrm{~K}$ (not shown).

The Peng-Robinson EOS ${ }^{58}$ is used to describe the properties of the single-phase fluid (the functions with superscript "EOS" in Sec. II). By using binary interaction parameters, the Peng-Robinson EOS accurately represents vapor-liquid equilibrium compositions for mixtures of hydrocarbons. ${ }^{59,60}$ The phase diagram of hexane-heptane at $T=298.15 \mathrm{~K}$ as calculated by the Peng-Robinson EOS is displayed in Fig. 3, where the binary interaction parameter is provided in Table II. The figure shows that VLE can be found between the saturation pressure of pure hexane $(\sim 20 \mathrm{kPa})$ and of pure heptane $(\sim 6 \mathrm{kPa})$, with dew points represented by the red solid curve and bubble points represented by the blue dashed curve.

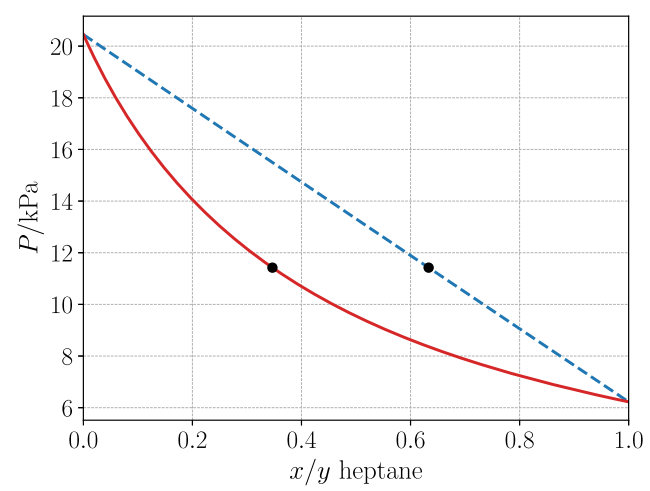

FIG. 3. Dew curve (red) and bubble curve (blue) of the hexane-heptane mixture at $298.15 \mathrm{~K}$ in pressure-composition space, with a VLE state marked (black points). Here $x$ and $y$ denote the mole fraction of heptane in the liquid and vapor phase, respectively. In Pxy-space, a planar density profile corresponds to one bubble point and one dew point; the corresponding plot in $\boldsymbol{\mu}$-space is qualitatively similar to Fig. 1 . 
TABLE II. Parameters used to generate results. Component 1 is hexane; component 2 is heptane. $\kappa_{1}$ and $\kappa_{2}$ are the pure-component influence parameters; $\kappa_{12}$ is the cross-influence parameter, while $k_{12}$ is the interaction parameter in the EOS mixing rule.

\begin{tabular}{lc}
\hline \hline Parameter & Value \\
\hline$k_{12}$ & -0.0011 \\
Temperature & $298.15 \mathrm{~K}$ \\
$\kappa_{1}$ & $4.18 \times 10^{-19} \mathrm{Jm}^{5} / \mathrm{mol}^{2}$ \\
$\kappa_{2}$ & $5.76 \times 10^{-19} \mathrm{Jm}^{5} / \mathrm{mol}^{2}$ \\
$\kappa_{12}$ & $\sqrt{\kappa_{1} \kappa_{2}}$ \\
\hline \hline
\end{tabular}

The entries of the influence matrix $\boldsymbol{K}$ were fitted to the pure-component surface tension values reported by Jasper ${ }^{61}$ at $300 \mathrm{~K}$, with the resulting values shown in Table II, and the cross-influence parameter $\kappa_{12}$ was described by the geometric mean rule. SGT then predicts the planar surface tension of the hexane-heptane mixture to vary nearly linearly with the liquid mole fraction of heptane. This is expected, as hexane and heptane are similar fluids.

\section{A. An explanation for the path dependence of the coefficients in the multicomponent Helfrich expansion}

As explained previously, the Tolman length and rigidities of a multicomponent fluid depend on the path taken through thermodynamic variable space. This becomes especially clear by considering Fig. 4, which presents a phase diagram in the chemical-potential space, where all coordinates that are not on the VLE envelope, $\mathcal{S}$ (thick solid line), represent either bubbles or droplets. The figure illustrates two paths from $\mathcal{S}$ to a uniquely defined spherical droplet state characterized by $\boldsymbol{\mu}^{\mathrm{s}}$, where the VLE states $A$ and $B$ have different liquid-phase compositions, pressures, and surface tensions. Both paths traverse

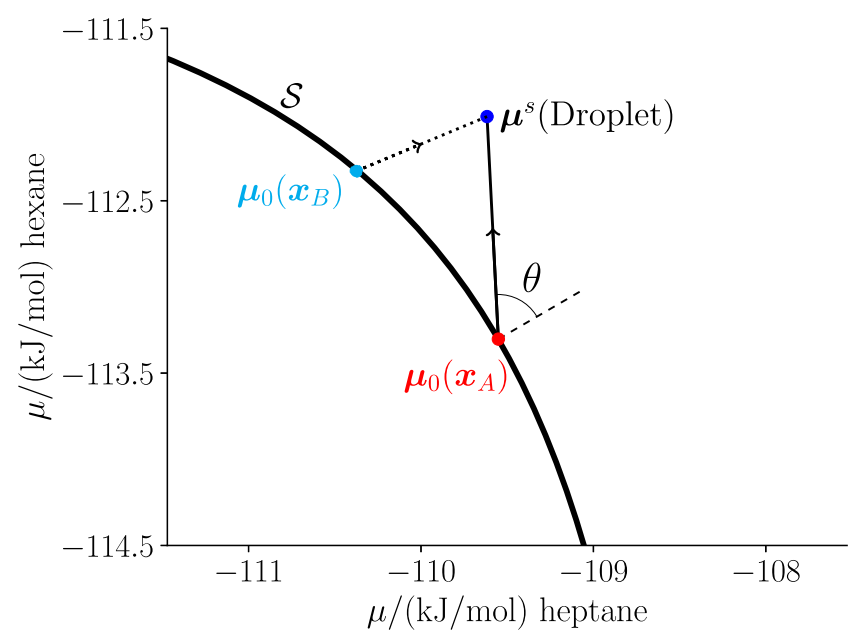

FIG. 4. A phase diagram of the chemical potential space, where all coordinates that are not on the VLE curve, $\mathcal{S}$ (thick solid line), represent either spherical bubbles or droplets of different compositions and radii. The thermodynamic properties of a droplet at $\boldsymbol{\mu}^{\mathrm{s}}$ (blue point) can be represented by a curvature expansion either along a path from State $A$ (red point and solid line) or from State $B$ (light blue point and dotted line). Here, $\boldsymbol{x}$ is the liquid-phase composition, which is different in the two VLE states. The path from State $A$ can be specified by the angle $\theta$, measured counterclockwise relative to the perpendicular (dashed curve) of $\mathcal{S}$ at $\boldsymbol{\mu}_{0}\left(\boldsymbol{x}_{A}\right)$. continuously through droplet states and are equally valid from a thermodynamic point of view. Given that the surface tension of the droplet, $\sigma^{\mathrm{s}}$, is accurately represented by the Helfrich expansion, the following relation is true:

$$
\sigma_{0}\left(x_{A}\right)\left(1-\frac{2 \delta_{A}}{R}\right)+\frac{k_{s, A}}{R^{2}}=\sigma_{0}\left(x_{B}\right)\left(1-\frac{2 \delta_{B}}{R}\right)+\frac{k_{s, B}}{R^{2}},
$$

where $x$ is the mole fraction in the liquid phase, the lefthand-side represents the Helfrich expansion from State $A$ and the right-hand-side is the Helfrich expansion from State $B$. Since $\sigma_{0}\left(x_{A}\right) \neq \sigma_{0}\left(x_{B}\right)$, Eq. (61) shows that the Helfrich coefficients of a multicomponent fluid depend on the VLE state used in the Helfrich expansion. The Tolman length and rigidities of a multicomponent fluid are thus only state functions after the path along which droplets and bubbles are considered has been specified. The rigidities also need the choice of dividing surface to be specified in order to be state functions.

\section{B. Behavior of the Helfrich coefficients from a fixed VLE state along different paths in thermodynamic state space}

The path from a VLE state along which droplets and bubbles are considered can for a binary mixture be characterized by the angle $\theta$ which measures the counterclockwise angle relative to the perpendicular of $\mathcal{S}$ at $\mu_{0}$ (see Fig. 4). For a fixed VLE state, and a given choice of the dividing surface, the Helfrich coefficients can thus be represented as functions of the angle $\theta$. We now fix the VLE state according to the specification in Table III, choose the total equimolar dividing surface and examine the dependence of the Tolman length and the rigidities on $\theta$. Figure 5 illustrates this dependence for the Tolman length.

A striking feature of the Tolman length in a multicomponent fluid is that it can take any real value, as illustrated by the red solid curve. However, the directions parallel to $\mathcal{P}$ are not meaningful for a curvature expansion of the surface tension since the curvature is constant in these directions, equal to zero. This is reflected in asymptotes in the Tolman length profile at $\theta= \pm \pi / 2$. The Tolman length changes sign somewhere in between, but it is negative for most values of $\theta$, including the perpendicular direction corresponding to $\theta=0$. Moreover, Fig. 5 shows that $\left\|\boldsymbol{\mu}_{1}\right\|$ approaches $\infty$ when $\theta$ approaches $\theta= \pm \pi / 2$ and has the symmetry of an even function with respect to $\theta$, with a minimum at $\theta=0$. This means that the perpendicular direction in $\boldsymbol{\mu}$ space $(\theta=0)$ is the one in which the chemical potential of large droplets is the least sensitive with respect to changes in curvature.

TABLE III. A VLE state (planar interface) and a droplet state (spherical interface) used to generate results. The VLE state is that shown in Figs. 3 and 4, and the droplet state is shown in Fig. 4. The mole fraction $x$ is that of heptane.

\begin{tabular}{lllcccc}
\hline \hline State & $P^{\text {vap }}(\mathrm{kPa})$ & $x^{\text {vap }}$ & $P^{\text {liq }}(\mathrm{kPa})$ & $x^{\text {liq }}$ & $R_{\mathrm{e}}(\mathrm{nm})$ & $R_{t}(\mathrm{~nm})$ \\
\hline VLE & 11.4 & 0.35 & 11.4 & 0.63 & $\infty$ & $\infty$ \\
Droplet & 16.50 & 0.49 & 3739.14 & 0.23 & 10.00 & 10.14 \\
\hline \hline
\end{tabular}




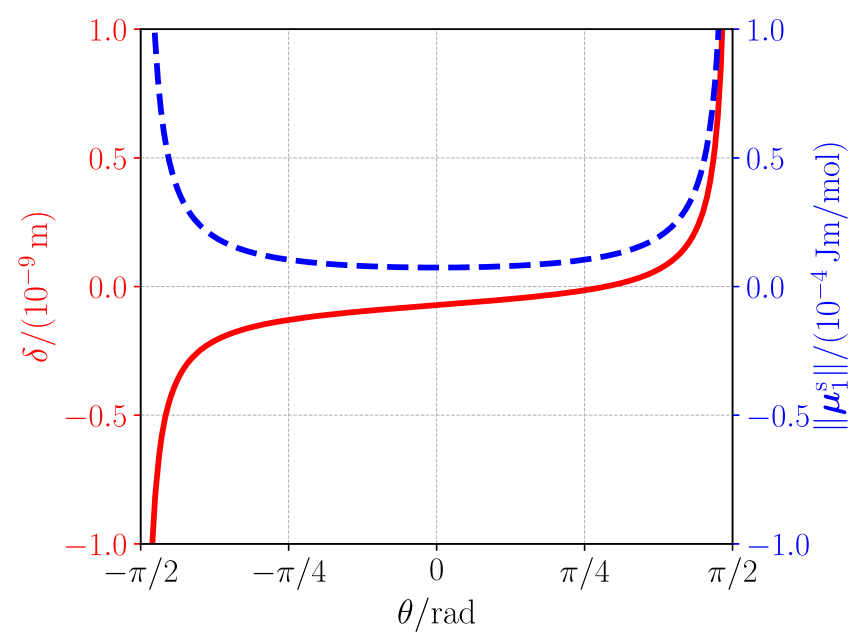

FIG. 5. The Tolman length $\delta$ (red curve) and $\left\|\boldsymbol{\mu}_{1}^{\mathrm{s}}\right\|$ (blue dashed curve) as functions of the direction in chemical-potential space, as measured by $\theta$.

Figure 6(a) shows how the second-order curvature correction for droplets and bubbles, called the spherical rigidity $k_{\mathrm{s}}=2 k+\bar{k}$, and $\boldsymbol{\mu}_{2}^{\mathrm{s}}$ vary with the choice of path. We have here considered a straight line in the chemical-potential space, implying that $\boldsymbol{\mu}_{1} \propto \boldsymbol{\mu}_{2}$. The spherical rigidity varies little for directions close to the normal direction, but starts to diverge at approximately $|\theta|>\pi / 4$.

We have also considered paths that cannot be described by straight lines in $\boldsymbol{\mu}$-space, but curves (like the path $\mathcal{P}_{b}$ in Fig. 1). To this end, we fixed the angle of $\mu_{1}$ to $\theta=0$ and varied the angle $\theta_{2}$ between $\boldsymbol{\mu}_{2}$ and the normal vector of the saturation locus; $\boldsymbol{\mu}_{2}$ is then fully determined by Eq. (15). The resulting behavior of $k_{\mathrm{s}}$ and $\left\|\mu_{2}\right\|$ is shown in Fig. 6(b), which reveals that the second-order coefficients are essentially constant when $\mu_{1}$ is fixed to the perpendicular direction. More generally, we find that for fixed directions of $\boldsymbol{\mu}_{1}$ close to the perpendicular direction, approximately $|\theta| \lesssim \pi / 6$, the rigidity constants are to a good approximation independent of $\theta_{2}$. We will therefore in the following constrain the discussion to paths that can be described by straight lines in $\boldsymbol{\mu}$-space.

\section{Behavior of the Helfrich coefficients across the phase envelope for fixed choices of paths}

The Tolman length and rigidities of a multicomponent fluid are only state functions and useful from a practical point of view after a path has been specified, as explained in Sec. IV A. Therefore, we next fix the path according to different choices and study the change in the Tolman length and rigidities along the phase envelope. We parametrize the phase envelope by using the liquid mole fraction of heptane.

The Tolman length exhibits a very different behavior across the phase envelope depending on the path chosen, as shown in Fig. 7(a). If the path is chosen so as to keep the composition at the center of the droplet constant (blue solid curve), then the Tolman length increases monotonically and close to linearly with the liquid mole fraction of heptane. If the vapor composition is kept constant, however (green dotted curve), the Tolman length exhibits a maximum. A similar behavior is seen if the path increases the chemical potential of hexane twice as much as heptane; this corresponds to paths that are straight lines in $\boldsymbol{\mu}$-space with a slope of 2 so that $\boldsymbol{\mu}_{1} \propto[1,2]$ (red curve). (The direction $[1,2]$ was chosen arbitrarily as an example.) When the path is perpendicular to the phase envelope in $\boldsymbol{\mu}$-space (black dashed curve), the Tolman length exhibits both a maximum and minimum. A common denominator for all the choices of paths in Fig. 7(a) is that they recover the pure-component Tolman lengths in the pure-component limit. This is in agreement with the consistency criteria listed in Sec. III C.

Unlike the path for which $\boldsymbol{\mu}_{1} \propto[1,2]$, the directions of $\mu_{1}$ for the other three paths have to be calculated. To calculate the perpendicular direction in $\boldsymbol{\mu}$-space, first note that $\boldsymbol{\mu}-\boldsymbol{\mu}_{0}$ is perpendicular to $\mathcal{S}$ if and only if $\left(\boldsymbol{\mu}-\boldsymbol{\mu}_{0}\right) \mathrm{d} \boldsymbol{\mu}_{0}$ is

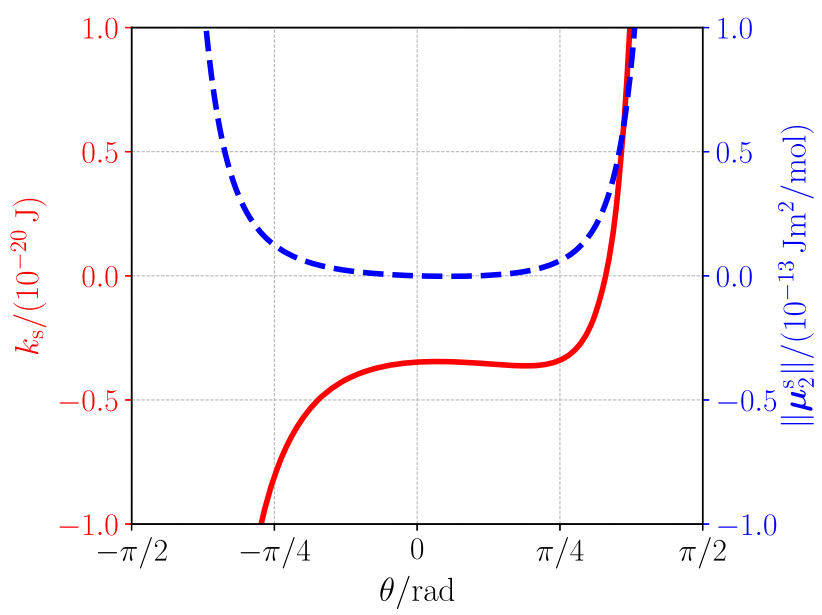

(a)

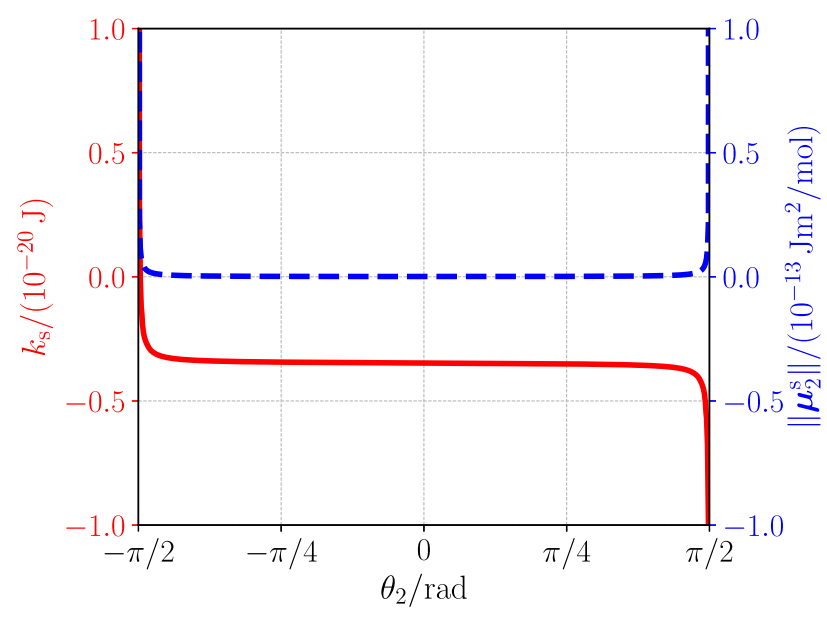

(b)

FIG. 6. The spherical rigidity $k_{s}=2 k+\bar{k}$ (red curve) and $\left\|\boldsymbol{\mu}_{2}^{\mathrm{s}}\right\|$ (blue dashed curve) as functions of the angle $\theta$, using the total equimolar radius. The angles have different meanings in the two plots: In (a) $\boldsymbol{\mu}_{1}^{\mathrm{s}} \propto \boldsymbol{\mu}_{2}^{\mathrm{s}}$ and $\boldsymbol{\theta}=\theta_{2}$. In (b) we have fixed $\boldsymbol{\mu}_{1}$ to be in the perpendicular direction $(\theta=0)$, while $\boldsymbol{\mu}_{2}$ forms an angle $\theta_{2}$ with the perpendicular direction. 


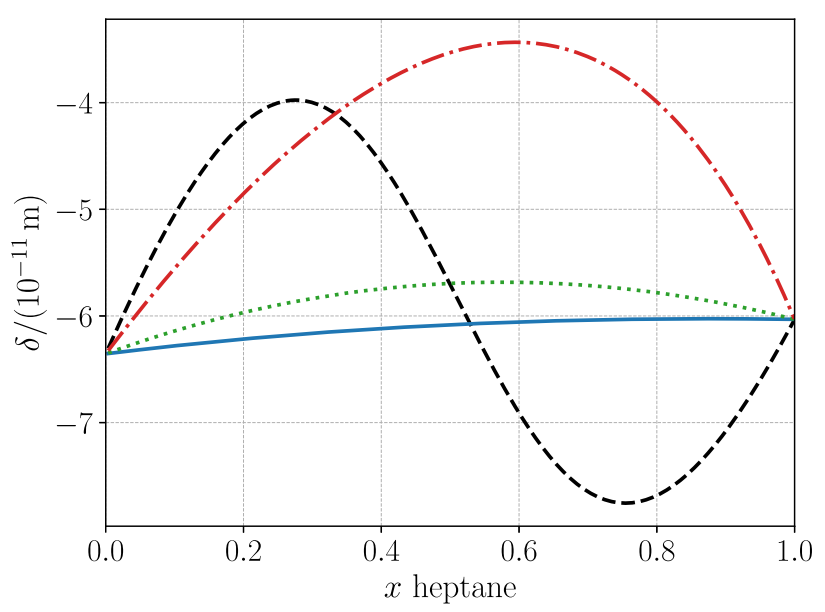

(a)

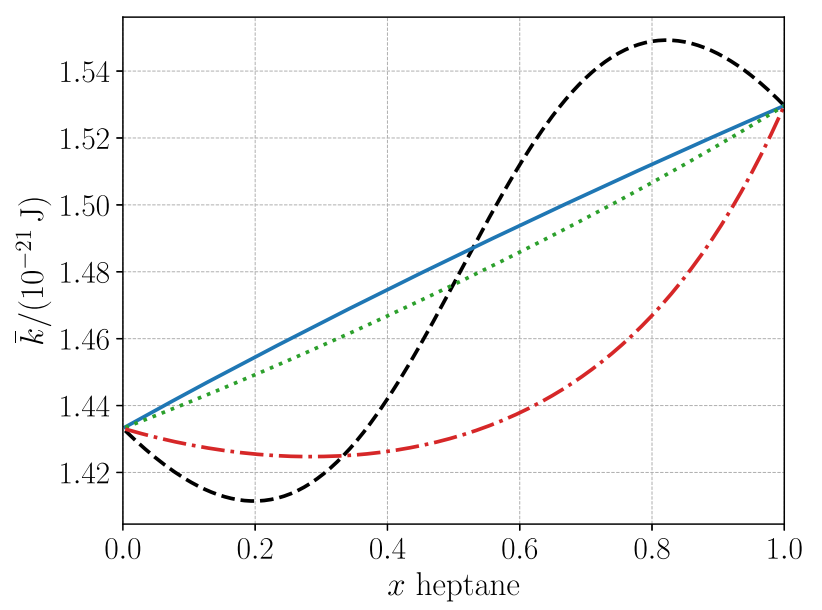

(c)

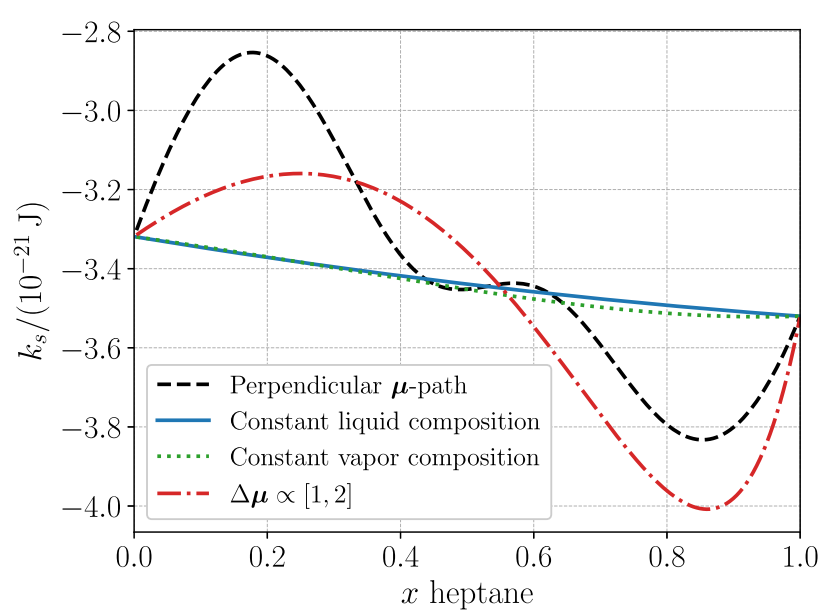

(b)

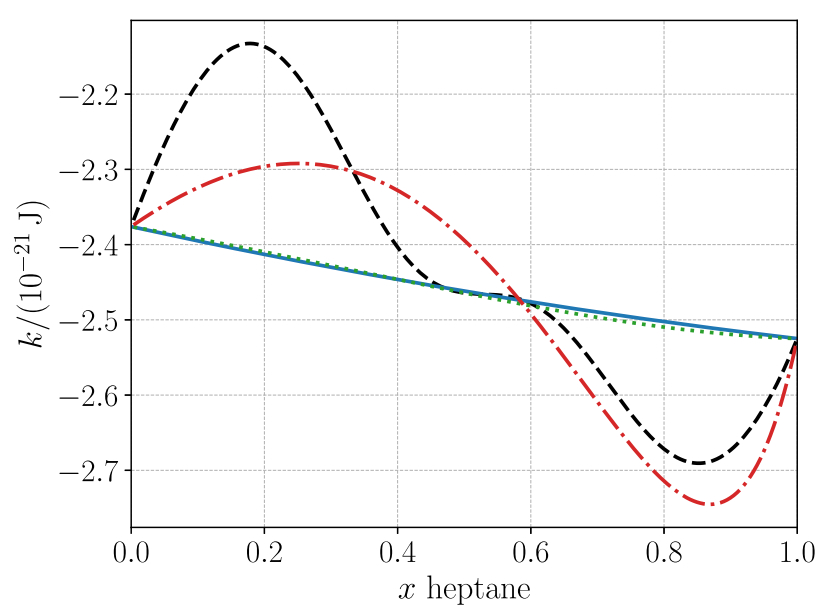

(d)

FIG. 7. Coefficients of the Helfrich expansion across the phase envelope at $T=298.15 \mathrm{~K}$ for the hexane-heptane mixture, using the total equimolar radius. The colors of the curves have the same meaning in all plots and is indicated in the legend in plot (b). (a) Tolman length $\delta$. (b) Spherical rigidity $k_{s}=2 k+\bar{k}$. (c) Gaussian rigidity $\bar{k}$. (d) Bending rigidity $k$.

zero for all vectors $\mathrm{d} \boldsymbol{\mu}_{0}$ that are tangent to $\mathcal{S}$ at $\boldsymbol{\mu}_{0}$. Since the Gibbs-Duhem relation shows that $\mathcal{S}$ is locally defined by the relation $\left(\rho_{0}^{\text {liq }}-\rho_{0}^{\text {vap }}\right) \mathrm{d} \mu_{0}=0$, it follows that the perpendicular directions in $\boldsymbol{\mu}$-space are proportional to $\left(\boldsymbol{\rho}_{0}^{\text {liq }}-\boldsymbol{\rho}_{0}^{\mathrm{vap}}\right)$. The calculation of the direction of $\boldsymbol{\mu}_{1}$ for paths of constant vapor or liquid composition is more involved and is described in the supplementary material.

A natural question to investigate is whether there exists any simple "mixing rule" for Tolman lengths, i.e., a formula that estimates the Tolman length of a mixture from pure-component values. Since the Tolman length is a pathdependent property, such mixing rules should be for the Tolman length along a particular path. After inspecting the selection of paths plotted in Fig. 7(a), it appears that, for this hydrocarbon mixture, the Tolman length $\delta$ along paths of constant liquid composition varies almost linearly with the liquid-phase composition; the same is true for the quantity $\sigma_{0} \delta$. For systems where this linearity holds, it is straightforward to implement curvature corrections for the surface tension as the only required information is the coefficients of the Helfrich expansion for the pure components and the composition of the liquid phase. We evaluate the accuracy of the curvature expansion along the constant-liquid-composition path in Secs. IV D and IV E.

We have also plotted the rigidity constants in Figs. 7(b)-7(d) for the case when $\boldsymbol{\mu}_{1} \propto \boldsymbol{\mu}_{2}$, corresponding to straight lines in $\boldsymbol{\mu}$-space. The behavior of these coefficients across the phase envelope is in general very different depending on the choice of path. However, for the paths corresponding to constant (to first order) liquid or vapor composition, we see that similar to the Tolman length, the rigidity constants depend nearly linearly on the liquid-phase composition. We conclude that, for the hexane-heptane mixture, all coefficients in the Helfrich expansion exhibit a nearly linear dependence on the liquid mole fraction of heptane for the path corresponding to constant liquid composition (solid curves), but for other choices of paths they generally vary in a less regular way. The value of the spherical rigidity constant $k_{s}$ is about $-1 k_{B} T$.

\section{Which path yields the most accurate representation of small droplets and bubbles?}

In Secs. IV B and IV C, we showed that the magnitude and the behavior of the Tolman length and the rigidities depend 
strongly on the path taken in the thermodynamic variable space. Figure 4 shows that the same droplet can be described by traversing different paths. It is important to emphasize that once the choice of dividing surface has been specified, the surface tension of a droplet is a state function and different paths will recover this surface tension within the accuracy of the Helfrich expansion. An important question then arises: which path yields the most accurate representation of droplets and bubbles? Using SGT as a reference, this accuracy can be determined by numerically evaluating the deviation between the second-order expansions in Eqs. (8)-(12) and the corresponding thermodynamic properties obtained from the full SGT representation of droplets.

One path of particular interest is the perpendicular path in chemical-potential space, as for a given droplet $\boldsymbol{\mu}_{\text {drop }}$ this corresponds to performing a curvature expansion from the closest VLE state $\boldsymbol{\mu}_{0}$, where closest means that $\left\|\boldsymbol{\mu}-\boldsymbol{\mu}_{0}\right\|$ is minimized. Figure 8 shows the relative error between the Helfrich expansion and the surface tension for the fixed droplet state specified in Table III. The figure shows that for all choices of paths satisfying $|\theta|<\pi / 4$, i.e., directions that are not too far away from the perpendicular direction in $\boldsymbol{\mu}$-space, the Helfrich expansion gives a very accurate representation of the surface tension, with a relative error below $0.1 \%$.

Figure 8 displays the accuracy for only one droplet; a more comprehensive evaluation should consider a wide range of droplets of varying composition. The bar charts in Fig. 9 show the absolute average relative deviation (AARD) between the second-order curvature expansion and the full SGT representation for droplets and bubbles of radius $10 \mathrm{~nm}$. By calculating the full SGT solution for 50 VLE states along the phase envelope, 50 bubbles, and 50 droplets, the deviation is calculated by estimating the $\mu, \sigma$, and $\Delta P$ for every droplet and bubble from every VLE state using a curvature expansion, and the AARD is plotted as a function of the counterclockwise angle $\theta$ measured with respect to the perpendicular direction. The results have been binned into 11 intervals of equal width for the angle $\theta$. Droplets corresponding to

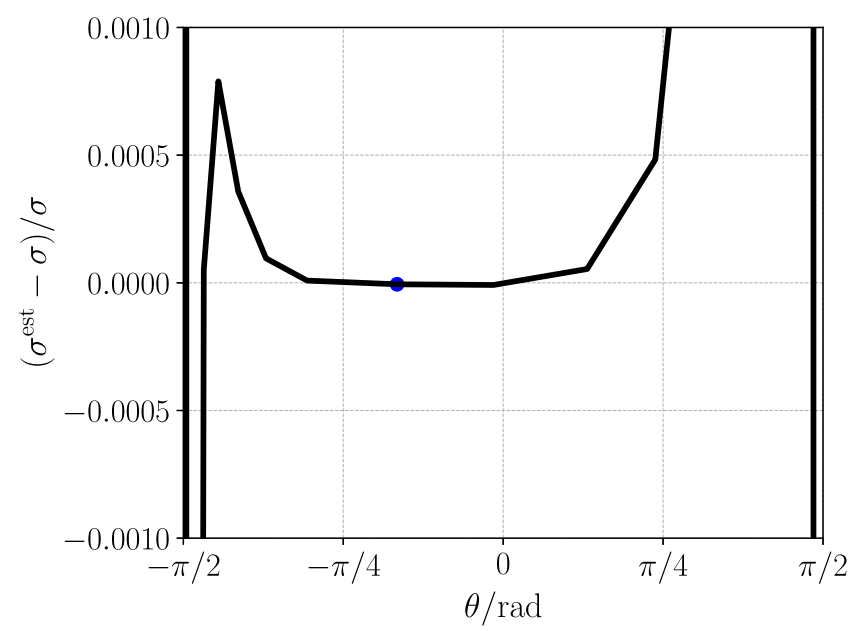

FIG. 8. The relative deviation when estimating the surface tension of the droplet in Table III from different VLE states, corresponding to different angles $\theta$, using the Helfrich expansion. The relative deviation for the path of constant liquid composition is $-6 \times 10^{-6}$ and is given by the blue point. $|\theta|>\pi / 2$ relative to a VLE state have not been considered. For the hexane-heptane mixture, Fig. 9 presents evidence that the perpendicular direction $\theta=0$ is, on average, the best choice of all fixed- $\theta$ paths. However, it is not so that the perpendicular direction is always the most accurate, and the minimum error for the quantities examined is usually shifted slightly toward $\theta>0$ for some droplet/bubble-states and toward $\theta<0$ for other droplet/bubble-states.

For the hexane-heptane mixture, it turns out that there is a path that is even more accurate on average that the perpendicular path $(\theta=0)$, namely, the one corresponding to constant liquid composition. The deviations for this path are given by the green dashed lines in Fig. 9.

In Table IV, we present the calculated AARDs for 50 droplets with even smaller radii, namely $5 \mathrm{~nm}$. It is clear that in the collective estimation of chemical potentials, surface tensions, and liquid-vapor pressure differences, the path of constant liquid composition is better than the perpendicular direction in $\boldsymbol{\mu}$-space, although for the surface tensions they give very similar results.

\section{E. How small droplets and bubbles can be accurately represented by the Helfrich expansion?}

For possible applications, it is important to know the size range for droplets and bubbles where the Helfrich expansion gives an accurate representation of the surface tension. In Fig. 10, we consider the fixed VLE state of Table III and compare the Helfrich expansion to the full SGT solution for droplets and bubbles of decreasing radius for different choices of $\theta$. For the perpendicular direction displayed in Fig. 10(a), a visual inspection shows that one has to consider the last bullet point before the Helfrich expansion (solid curve) starts to deviate significantly from the center location of the surface tension predicted by SGT, i.e., at $R \sim 2.5 \mathrm{~nm}$. The accuracy of the path of constant liquid composition is displayed in Fig. 10(b) and is seen to be very similar to that of the perpendicular direction; this is consistent with Figs. 9(c)-9(d) and Table IV. For these paths, the Helfrich expansion gives the same predictions of the surface tension as the full SGT to a very high accuracy, even for droplets with radii of less than $3 \mathrm{~nm}$.

A larger deviation can be seen in Fig. 10(c), corresponding to $\theta=5 \pi / 18$, while Fig. $10(\mathrm{~d})$ shows how choosing $|\theta|$ too large results in useless predictions with the Helfrich expansion. Unlike the other directions in Fig. 10, the direction $\theta=5 \pi / 12$ has a positive Tolman length, and moreover a positive spherical rigidity. The second-order derivative of $\sigma$ with respect to $R^{-1}$ is seen to change sign from positive for small curvatures to negative at large curvatures, and the second-order (Helfrich) expansion is in fact worse than the first-order (Tolman) expansion since it predicts that the surface tension diverges to infinity. We have not plotted the bubble solutions in Fig. 10(d) since there is a limit to how small bubbles that can exist along in directions in $\boldsymbol{\mu}$-space that are almost tangent to the phase envelope. This is because a straight path in the bubble regime may lead us back to the droplet regime again (imagine changing the direction of the path $\mathcal{P}_{A}$ in Fig. 1 to be almost tangential to $\mathcal{S}$ ). 


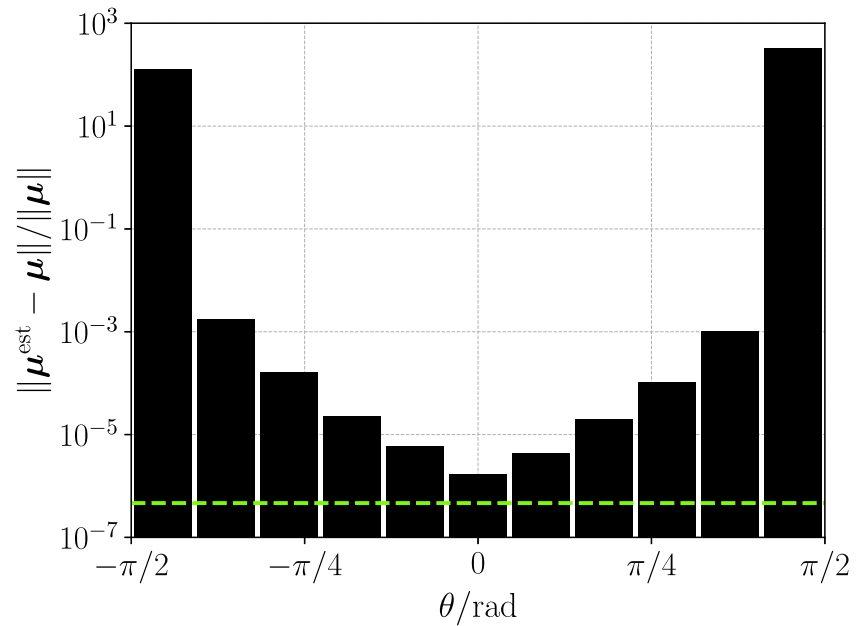

(a)

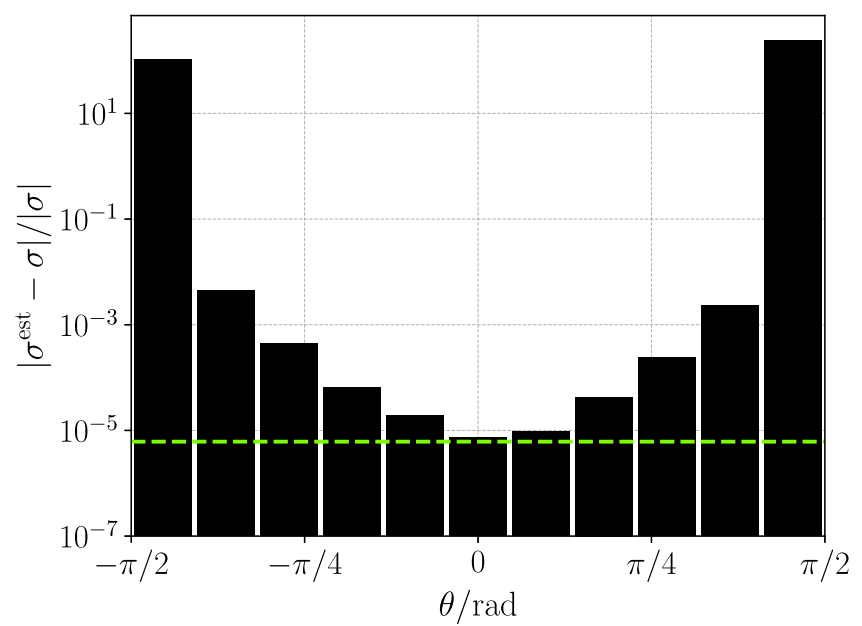

(c)

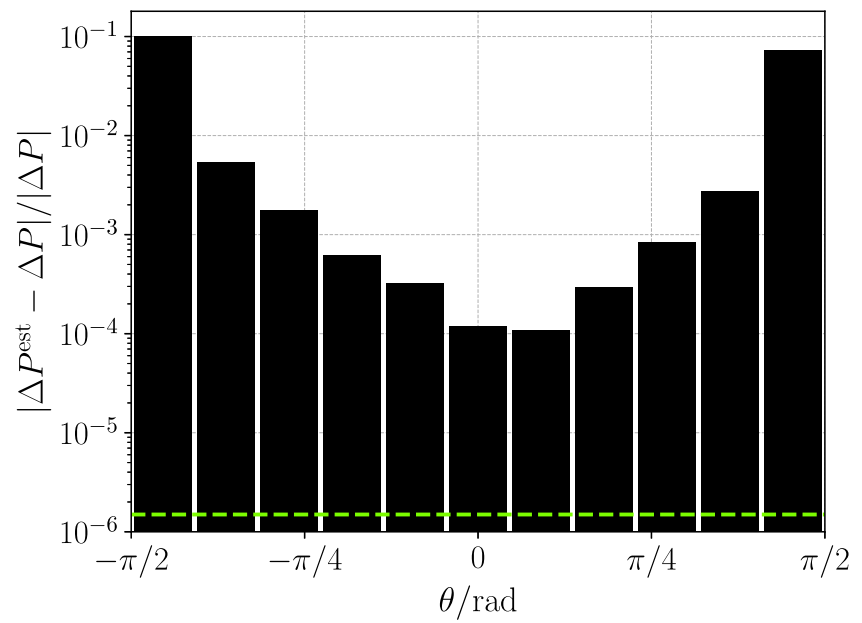

(e)

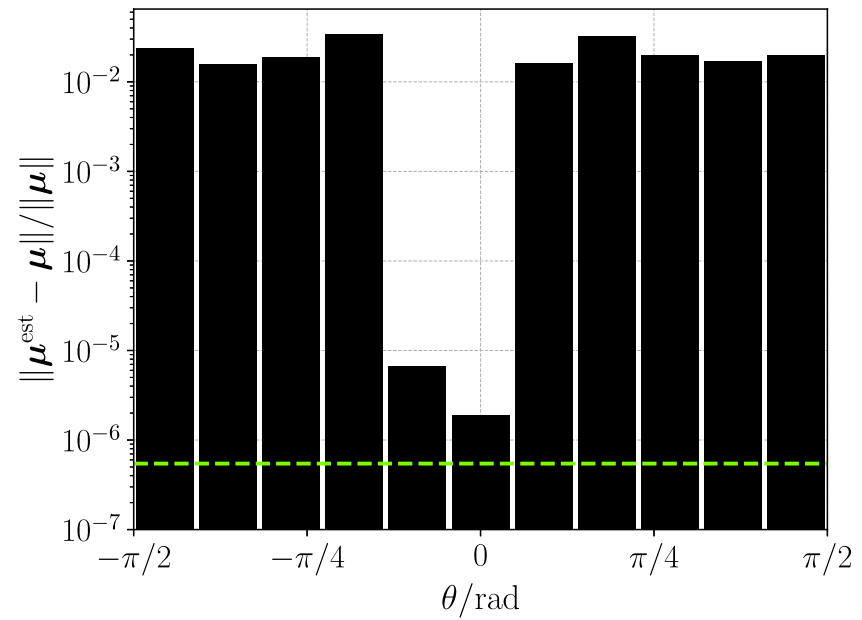

(b)

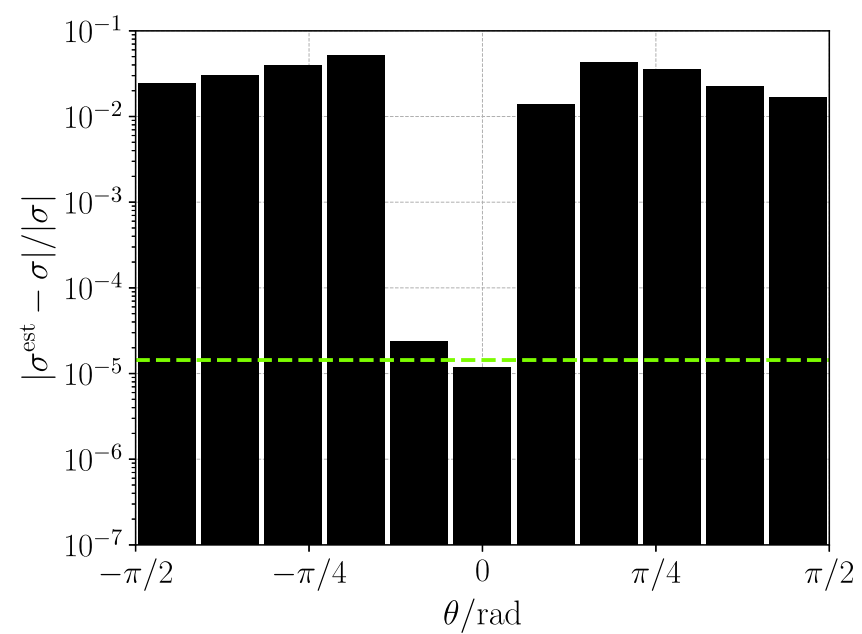

(d)

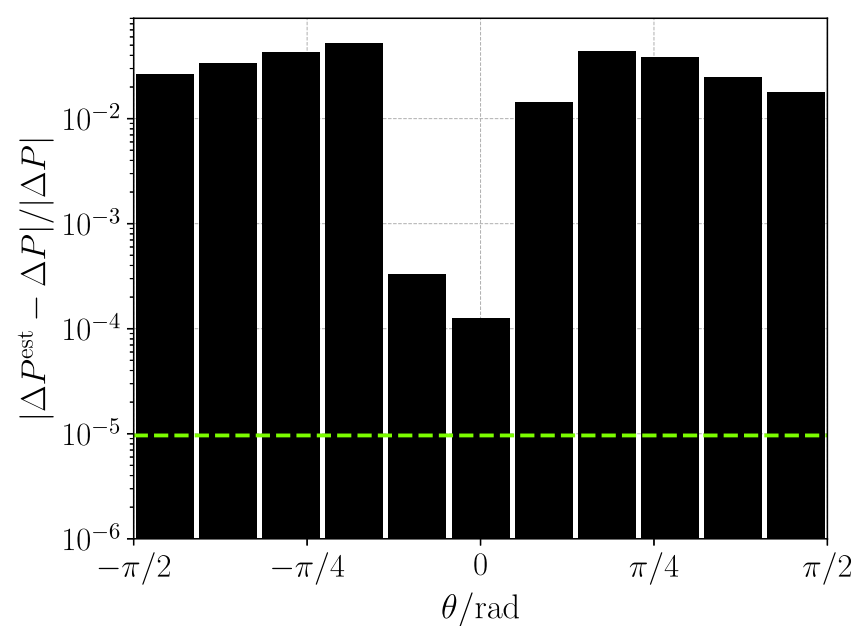

(f)

FIG. 9. The AARD of thermodynamic properties estimated using the curvature expansion for droplets (left-hand side) and bubbles (right-hand side) with radius $10 \mathrm{~nm}$. The black bars represent deviations as a function of $\theta$, while the green dashed line is the deviation for the constant liquid composition direction. (a) Relative error in estimating $\boldsymbol{\mu}$ for a droplet. (b) Relative error in estimating $\boldsymbol{\mu}$ for a bubble. (c) Relative error in estimating $\sigma$ for a droplet. (d) Relative error in estimating $\sigma$ for a bubble. (e) Relative error in estimating $\Delta P$ for a droplet. (f) Relative error in estimating $\Delta P$ for a bubble.

While Figs. 10(c) and 10(d) demonstrate how a poor choice of path impacts the accuracy, we also mention another pitfall that must be avoided when considering paths $\mathcal{P}$ that cross the VLE envelope so that the curvature changes sign. If the path $\mathcal{P}$ is not smooth across the envelope, the curvature coefficients will have different values when estimated from 
TABLE IV. Average absolute relative deviations when estimating the thermodynamic properties of droplets with radius $5 \mathrm{~nm}$ and varying compositions at $298.15 \mathrm{~K}$, using various curvature expansions. The deviations are calculated by comparing the values predicted from the curvature expansion to that of the full SGT description of the droplets.

\begin{tabular}{lccc}
\hline \hline Expansion type & $\left\|\mu^{\text {est }}-\mu\right\| /\|\mu\|$ & $\left|\sigma^{\text {est }}-\sigma\right| /|\sigma|$ & $\left|\Delta P^{\text {est }}-\Delta P\right| /|\Delta P|$ \\
\hline Linear mixing rules & $6.2 \times 10^{-2}$ & $2.9 \times 10^{-4}$ & $3.2 \times 10^{-4}$ \\
Constant liquid composition & $3.7 \times 10^{-6}$ & $3.0 \times 10^{-5}$ & $1.3 \times 10^{-5}$ \\
Perpendicular direction & $1.2 \times 10^{-5}$ & $4.4 \times 10^{-5}$ & $4.5 \times 10^{-4}$ \\
\hline
\end{tabular}

bubble properties compared to when they are estimated from droplet properties, and the curves plotted in Fig. 10 would then exhibit "kinks." This caveat applies equally well to nonisothermal variations of curvature, in which case the Helfrich coefficients acquire a path-dependence even for onecomponent systems, and has previously been a source of confusion in the literature. ${ }^{62}$ The paths $\mathcal{P}_{A}$ and $\mathcal{P}_{B}$ in Fig. 1 are examples of smooth paths.

\section{F. Practical use of the Helfrich expansion for mixtures}

At least for describing droplets and bubbles of the hexaneheptane mixture, there is one choice of path that stands out: the path of constant liquid composition. On average, it yields the most accurate representation of the surface tension with the Helfrich expansion. In addition, the Tolman length and rigidities of the mixture are nearly linear in the mole fraction of the liquid phase. In order to use the Helfrich coefficients, for example, to describe the critical cluster/cavity in nucleation, the only requirement would be the pure component values of the curvature expansion coefficients and the liquid-phase composition.

We have tested the applicability of the linear mixing rules that apply to the path of constant liquid-phase composition by obtaining the curvature expansion coefficients for the singlecomponent systems, and then assuming a linear dependence with respect to the mole fraction at the center of the droplet, this corresponds to assuming that $\sigma_{0}, \sigma_{0} \delta$, and $k_{s}$ are linear in the liquid composition. The results are shown in the second row of Table IV. The table shows that for this hydrocarbon mixture, the linear mixing rule works very well for estimating the surface tension and the pressure difference. Results for the estimation of the droplets' chemical potential are less satisfactory, but usually also of less interest. The average accuracy for the surface tension of $5 \mathrm{~nm}$ droplets as predicted by the Helfrich expansion with linear mixing rules is $0.03 \%$.

These conclusions remain valid at higher temperatures. The good performance of the linear mixing rule is not expected to hold for all mixtures, and may be a consequence of the fact that for the hexane-heptane mixture the planar surface tension

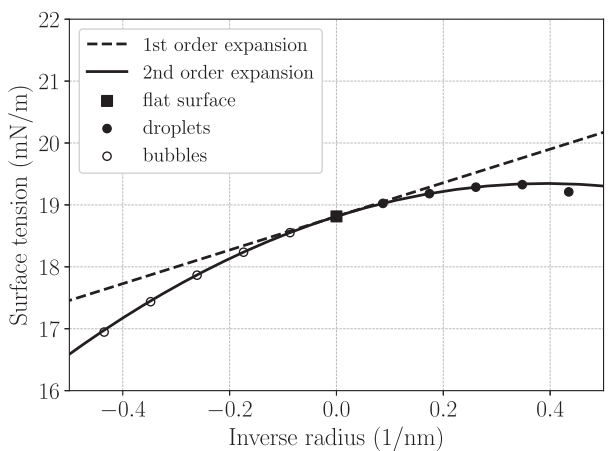

(a)

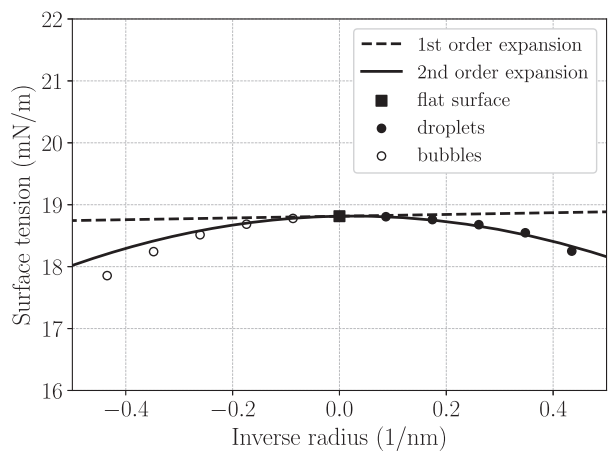

(c)

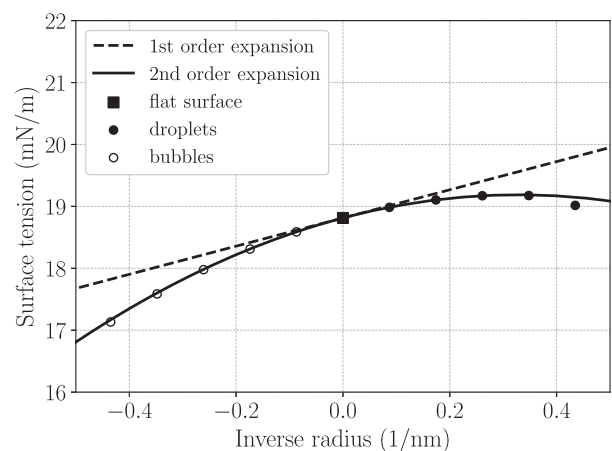

(b)

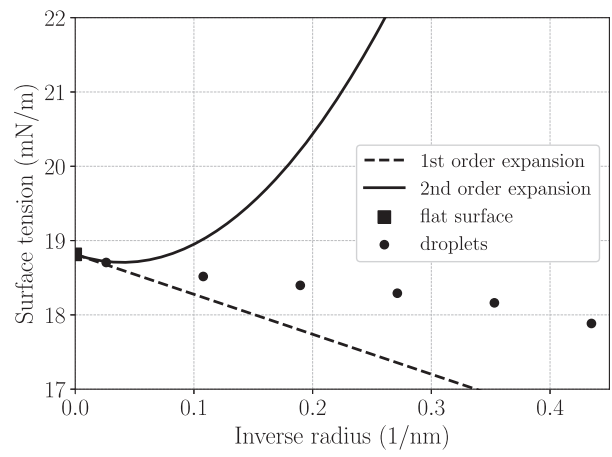

(d)
FIG. 10. Accuracy of the curvature expansion from a fixed VLE state as a function of inverse radius $1 / R_{\mathrm{e}}$, where $R_{\mathrm{e}}$ is the total equimolar radius. All plots correspond to straight paths through $\boldsymbol{\mu}$ space, forming an angle $\theta$ to the perpendicular direction of the VLE envelope $\mathcal{S}$, as illustrated in Fig. 4. The VLE state (flat surface) in these plots is specified in Table III. (a) Perpendicular path, $\theta=0$. (b) Direction of constant liquid composition, $\theta=-11.5^{\circ}$. (c) $\theta=5 \pi / 18=50^{\circ}$. (d) $\theta=5 \pi / 12=75^{\circ}$. 
is approximately linear in the liquid composition. Although the linear rule for planar surface tension is known to fail for many mixtures ${ }^{63}$ our results demonstrate that at least for some mixtures it is possible to develop approximate mixing rules for the coefficients in the Helfrich expansion. For mixtures where the linear mixing rule fails, one could try to extend the established non-linear mixing rules ${ }^{63}$ for planar surface tension to curved surfaces.

\section{CONCLUSION}

In this work, we have presented and explained the general theory for the curvature dependence of the surface tension for multicomponent fluids by use of the Helfrich expansion. Square gradient theory (SGT) was employed for a mixture of hexane-heptane at $298.15 \mathrm{~K}$ to obtain the quantitative estimates of the first-order correction, given by the Tolman length, and the second-order corrections, given by the rigidities. With these coefficients, the Helfrich expansion can be used to describe the surface tension of spherical or cylindrical droplets/bubbles or of arbitrarily curved vapor-liquid films up to second-order in the curvature. The Tolman length of a multicomponent fluid was shown to be independent of the choice of dividing surface. The rigidities do depend on the choice of dividing surface, and we presented simple formulae to account for the change in the rigidities with a change of dividing surface.

Unlike in the one-component case, the Tolman length and rigidities of a multicomponent fluid depend on the path taken through thermodynamic variable space, i.e., how the pressure and composition of the exterior phase are changed as the droplet/bubble shrinks and the curvature increases. We explained where this path dependence comes from and showed that the magnitude and behavior of the Tolman length and rigidities depend strongly on the choice of path. The path that on average gave the most accurate representation of small droplets and bubbles was found to be the one corresponding to constant liquid composition; however, good accuracy was found for all paths in directions sufficiently far away from the directions parallel to the saturation locus, i.e., sufficiently close to the perpendicular direction in $\boldsymbol{\mu}$-space.

All the paths considered reproduce the pure-component Tolman length and rigidities in the limit of pure hexane and heptane. For the hydrocarbon mixture investigated, we found that the Tolman length and rigidity constants are nearly linear in the mole fraction of the liquid phase along paths of constant liquid composition. We compared the Helfrich expansion to the full description of droplets and bubbles as provided by SGT, finding excellent agreement even for very small droplet/bubble sizes. For the path of constant liquid composition, the Helfrich expansion reproduces the surface tension from SGT within $0.1 \%$ for droplet radii down to 3 nanometers. Valuable future work would be to test the generality of our findings for other mixtures, and more sophisticated density functional theories than SGT. The multicomponent Helfrich expansion and the framework developed in this work have the potential to be used in several important applications, such as in nucleation theory, or in the description of multicomponent droplets forming on solid surfaces.

\section{SUPPLEMENTARY MATERIAL}

See supplementary material for details regarding the following: (1) the derivation of the transformation formulae for the Tolman length and rigidity constants upon a change of dividing surface; (2) the derivation of the curvature expansion of the excess grand free energy per surface area; (3) the calculation of the direction of $\boldsymbol{\mu}_{1}$ for paths of constant vapor or liquid composition.

\section{ACKNOWLEDGMENTS}

The authors thank Dick Bedeaux, Signe Kjelstrup, and David Reguera for helpful discussions.

\section{APPENDIX: SGT FORMULA FOR THE NOTIONAL DERIVATIVE OF A DROPLET}

Expanding the formula for the notional derivative, $[\partial \sigma / \partial R]_{T, \boldsymbol{\mu}}=\mathrm{d} \sigma / \mathrm{d} R+\boldsymbol{\Gamma} \mathrm{d} \boldsymbol{\mu} / \mathrm{d} R$, yields

$$
\begin{aligned}
{\left[\frac{\partial \sigma}{\partial R}\right]_{T, \boldsymbol{\mu}}=} & \frac{\sigma_{1}+\boldsymbol{\mu}_{1} \boldsymbol{\Gamma}_{0}}{R^{2}} \\
& -\frac{2 \sigma_{2}+\boldsymbol{\mu}_{1} \boldsymbol{\Gamma}_{1}+2 \boldsymbol{\mu}_{2} \boldsymbol{\Gamma}_{2}}{R^{3}}+\cdots .
\end{aligned}
$$

Using the the SGT expressions for $\sigma_{1}$ and $\sigma_{2}$ given by Eqs. (45) and (46), and the formulae for the adsorptions given by Eqs. (17) and (18), we find that

$$
\begin{aligned}
{\left[\frac{\partial \sigma}{\partial R}\right]_{T, \boldsymbol{\mu}}=} & -\frac{g}{R^{2}} \int\left(z \boldsymbol{\rho}_{0 z}^{\top} \boldsymbol{K} \boldsymbol{\rho}_{0 z}\right) \mathrm{d} z-\frac{g}{R^{3}} \int\left[(g-1) z^{2} \boldsymbol{\rho}_{0 z}^{\top} \boldsymbol{K} \boldsymbol{\rho}_{0 z}\right. \\
& \left.-\boldsymbol{\rho}_{0 z}^{\boldsymbol{\top}} \boldsymbol{K} \boldsymbol{\rho}_{1}+z^{2} \boldsymbol{\mu}_{1}^{\mathrm{s}} \boldsymbol{\rho}_{0 z}+z \boldsymbol{\mu}_{1}^{\mathrm{s}} \boldsymbol{\rho}_{0}^{\mathrm{E}}\right] \mathrm{d} z+\cdots .
\end{aligned}
$$

All the coefficients in this expansion are zero for the surface of tension. Setting the $\left(1 / R^{2}\right)$-coefficient to zero yields a compact characterization of the position of the surface of tension for a plane surface in SGT:

$$
\int z \boldsymbol{\rho}_{0 z}^{\top} \boldsymbol{K} \boldsymbol{\rho}_{0 z} \mathrm{~d} z=0
$$

Using the formulae for parallel and tangential pressures for the SGT model ${ }^{40}$ one can verify that Eq. (A1) is equivalent to requiring that the first moment of the parallel minus the tangential pressure to be zero. Since the surface tension equals the integral of the parallel minus the tangential pressure, the surface of tension is the effective position at which the surface tension acts.

Moreover, setting the $\left(1 / R^{3}\right)$-coefficient to zero yields an integral condition that $\boldsymbol{\rho}_{1}$-which depends on the choice of dividing surface - has to satisfy when computed relative to the surface of tension. This is most conveniently written in the form

$$
\int \boldsymbol{\rho}_{0 z}^{\top} \boldsymbol{K} \boldsymbol{\rho}_{1} \mathrm{~d} z=\int\left[(g-1) z^{2} \boldsymbol{\rho}_{0 z}^{\top} \boldsymbol{K} \boldsymbol{\rho}_{0 z}+z^{2} \boldsymbol{\mu}_{1}^{\mathrm{s}} \boldsymbol{\rho}_{0 z}+z \boldsymbol{\mu}_{1}^{\mathrm{s}} \boldsymbol{\rho}_{0}^{\mathrm{E}}\right] \mathrm{d} z
$$

\footnotetext{
${ }^{1} \mathrm{~S}$. Ono and S. Kondo, "Molecular theory of surface tension in liquids," in Structure of Liquids/Struktur der Flüssigkeiten (Springer, Berlin, Heidelberg, 1960), pp. 134-280.

${ }^{2}$ A. E. van Giessen and E. M. Blokhuis, J. Chem. Phys. 116, 302 (2002).
} 
${ }^{3}$ Y. A. Lei, T. Bykov, S. Yoo, and X. C. Zeng, J. Am. Chem. Soc. 127, 15346 (2005).

${ }^{4} \mathrm{Z}$. Li and J. Wu, Ind. Eng. Chem. Res. 47, 4988 (2008).

${ }^{5}$ A. E. van Giessen and E. M. Blokhuis, J. Chem. Phys. 131, 164705 (2009).

${ }^{6}$ B. J. Block, S. K. Das, M. Oettel, P. Virnau, and K. Binder, J. Chem. Phys. 133, 154702 (2010).

${ }^{7}$ A. Tröster, M. Oettel, B. Block, P. Virnau, and K. Binder, J. Chem. Phys. 136, 064709 (2012).

${ }^{8}$ M. Horsch, H. Hasse, A. K. Shchekin, A. Agarwal, S. Eckelsbach, J. Vrabec, E. A. Müller, and G. Jackson, Phys. Rev. E 85, 031605 (2012).

${ }^{9}$ R. C. Tolman, J. Chem. Phys. 17, 333 (1949).

${ }^{10}$ A. Malijevský and G. Jackson, J. Phys.: Condens. Matter 24, 464121 (2012).

${ }^{11}$ E. M. Blokhuis and A. E. van Giessen, J. Phys.: Condens. Matter 25, 225003 (2013).

${ }^{12} \emptyset$. Wilhelmsen, D. Bedeaux, and D. Reguera, J. Chem. Phys. 142, 064706 (2015).

${ }^{13}$ N. Bruot and F. Caupin, Phys. Rev. Lett. 116, 056102 (2016).

${ }^{14}$ A. Tröster and K. Binder, Phys. Rev. Lett. 107, 265701 (2011).

${ }^{15}$ W. Helfrich, Z. Naturforsch., C 28, 693 (1973).

${ }^{16}$ L. G. MacDowell, P. Llombart, J. Benet, J. G. Palanco, and A. GuerreroMartinez, ACS Omega 3, 112 (2018).

${ }^{17}$ J. Kim, I. Hwang, and B. Weon, Sci. Rep. 7, 42848 (2017).

${ }^{18}$ J. S. Rowlinson, J. Phys.: Condens. Matter 6, A1 (1994).

${ }^{19}$ J. Schmelzer, I. Gutzow, and J. J. Schmelzer, J. Colloid Interface Sci. 178, 657 (1996).

${ }^{20}$ P. Debenedetti, Metastable Liquids: Concepts and Principles (Princeton University Press, Princeton, 1996).

${ }^{21} \mathrm{H}$. Vehkamäki, Classical Nucleation Theory in Multicomponent Systems (Springer Verlag, Berlin, 2006).

${ }^{22}$ V. G. Baidakov, S. P. Protsenko, and V. M. Bryukhanov, Chem. Phys. Lett. 663, 57 (2016).

${ }^{23}$ A. K. Shchekin, T. S. Lebedeva, and D. V. Tatyanenko, Fluid Phase Equilib. 424, 162 (2016).

${ }^{24}$ S. R. Haqshenas, I. J. Ford, and N. Saffari, J. Chem. Phys. 145, 024315 (2016).

${ }^{25}$ T. Hiratsuka, H. Tanaka, and M. T. Miyahara, J. Phys. Chem. C 121, 26877 (2017).

${ }^{26}$ B. Cheng, G. A. Tribello, and M. Ceriotti, J. Chem. Phys. 147, 104707 (2017).

${ }^{27} \emptyset$. Wilhelmsen, D. Bedeaux, and D. Reguera, J. Chem. Phys. 142, 171103 (2015).

${ }^{28}$ M. N. Joswiak, R. Do, M. F. Doherty, and B. Peters, J. Chem. Phys. 145, 204703 (2016)

${ }^{29}$ K. Mishima, S. Nakamae, H. Ohshima, and T. Kondo, Chem. Phys. Lipids 110, 27 (2001).

${ }^{30}$ M. Khalkhali, N. Kazemi, H. Zhang, and Q. Liu, J. Chem. Phys. 146, 114704 (2017).

${ }^{31}$ M. Kanduč, J. Chem. Phys. 147, 174701 (2017).

${ }^{32}$ F. O. Koenig, J. Chem. Phys. 18, 449 (1950).
${ }^{33}$ J. Groenewold and D. Bedeaux, Phys. A 214, 356 (1995).

${ }^{34}$ E. Santiso and A. Firoozabadi, AIChE J. 52, 311 (2006).

${ }^{35}$ C. F. Weinaug and D. L. Katz, Ind. Eng. Chem. 35, 239 (1943).

${ }^{36}$ D. Macleod, Trans. Faraday Soc. 19, 38 (1923).

${ }^{37}$ J.-S. Li and G. Wilemski, J. Chem. Phys. 118, 2845 (2003).

${ }^{38}$ A. J.-M. Yang, J. Chem. Phys. 82, 2082 (1985).

${ }^{39}$ M. Iwamatsu and Y. Okabe, J. Chem. Phys. 133, 044706 (2010).

${ }^{40} \emptyset$. Wilhelmsen, D. Bedeaux, S. Kjelstrup, and D. Reguera, J. Chem. Phys. 140, 024704 (2014).

${ }^{41}$ E. M. Blokhuis and J. Kuipers, J. Chem. Phys. 124, 074701 (2006).

${ }^{42}$ E. M. Blokhuis, J. Groenewold, and D. Bedeaux, Mol. Phys. 96, 397 (1999).

${ }^{43}$ M. Napiórkowski and S. Dietrich, Phys. Rev. E 47, 1836 (1993).

${ }^{44} \mathrm{~J}$. Rowlinson and B. Widom, Molecular Theory of Capillarity (Clarendon Press, Oxford, 1984).

${ }^{45}$ J. Gibbs, "On the equilibrium of heterogeneous substances," in The Scientific Papers of J. Willard Gibbs (Ox Bow Press, London, 1993).

${ }^{46}$ J. Groenewold, "Statistical thermodynamics of surfaces and electrolyte depletion," Ph.D. thesis, Leiden University, 1997.

${ }^{47}$ V. Kalikmanov, Statistical Physics of Fluids (Springer Verlag, Berlin, 2011).

${ }^{48}$ J. S. Rowlinson, J. Stat. Phys. 20, 197 (1979).

${ }^{49} \mathrm{To}$ prove that $\boldsymbol{\rho}^{\mathrm{liq}}(\boldsymbol{\mu})$ a well-defined and differentiable function, note that $\boldsymbol{\mu}_{\rho}\left(\boldsymbol{\rho}^{\mathrm{liq}}\right)$ is the Hessian of the internal energy density of the liquid phase, and thus positive definite since the liquid is stable. The conclusion now follows from the inverse function theorem.

${ }^{50}$ E. M. Blokhuis and D. Bedeaux, Mol. Phys. 80, 705 (1993).

${ }^{51} \emptyset$. Wilhelmsen, T. T. Trinh, S. Kjelstrup, and D. Bedeaux, J. Phys. Chem. C 119, 8160 (2015).

${ }^{52} \emptyset$. Wilhelmsen, T. T. Trinh, A. Lervik, V. K. Badam, S. Kjelstrup, and D. Bedeaux, Phys. Rev. E 93, 032801 (2016).

${ }^{53}$ J. D. van der Waals, J. Stat. Phys. 20, 200 (1979).

${ }^{54}$ E. Jones, T. Oliphant, P. Peterson et al., SciPy: Open source scientific tools for Python, 2001, http://www.scipy.org/.

${ }^{55}$ Z. Qiao and S. Sun, SIAM J. Sci. Comput. 36, B708 (2014).

${ }^{56}$ X. Mu, F. Frank, F. O. Alpak, and W. G. Chapman, Fluid Phase Equilib. 435, 118 (2017).

${ }^{57}$ X. Liang and M. L. Michelsen, Fluid Phase Equilib. 451, 79 (2017).

${ }^{58}$ D. Y. Peng and D. B. Robinson, Ind. Eng. Chem. Fundam. 15, 59 (1976).

${ }^{59}$ G. M. Kontogeorgis and G. Folas, Thermodynamic Models for Industrial Applications (Wiley, 2010).

${ }^{60} \emptyset$. Wilhelmsen, A. Aasen, G. Skaugen, P. Aursand, A. Austegard, E. Aursand, M. A. Gjennestad, H. Lund, G. Linga, and M. Hammer, Ind. Eng. Chem. Res. 56, 3503 (2017).

${ }^{61}$ J. J. Jasper, J. Phys. Chem. Ref. Data 1, 841 (1972).

${ }^{62} \emptyset$. Wilhelmsen, D. Bedeaux, and S. Kjelstrup, Phys. Chem. Chem. Phys. 16, 10573 (2014).

${ }^{63}$ B. E. Poling, J. M. Prausnitz, and J. P. O' Connell, The Properties of Gases and Liquids, 5th ed. (McGraw-Hill, 2001). 